\title{
SUBDIVISIONS AND LOCAL $h$-VECTORS
}

\author{
RICHARD P. STANLEY
}

\section{PART I. SIMPLICIAL COMPLEXES}

\section{INTRODUCTION}

Let $\Delta$ be an (abstract) simplicial complex of dimension $d-1$ with $f_{i} i$ dimensional faces (or $i$-faces, for short). (For undefined terminology, see, e.g., $\left.\left[\mathrm{Sta}_{8}\right].\right)$ Throughout this paper, all simplicial complexes will be finite. The $f$ vector of $\Delta$ is given by $f(\Delta)=\left(f_{0}, \ldots, f_{d-1}\right)$, with the understanding that $f_{-1}=1$ unless $\Delta=\varnothing$. We will be concerned here with properties of the $f$ vector of subdivisions of $\Delta$. It is often more convenient to deal not with the $f$-vector, but rather the $h$-vector $h(\Delta)=\left(h_{0}, \ldots, h_{d}\right)$, defined by

$$
\sum_{i=0}^{d} f_{i-1}(x-1)^{d-i}=\sum_{i=0}^{d} h_{i} x^{d-i} .
$$

For instance, if $\Delta$ is a Cohen-Macaulay complex [ $\mathrm{Sta}_{4} ; \mathrm{Sta}_{8}$, Chapter II.3], then $h_{i} \geq 0$ (and in fact $h(\Delta)$ can be completely characterized in an elegant way). We often deal not with the $h$-vector $h(\Delta)=\left(h_{0}, h_{1}, \ldots, h_{d}\right)$ per se, but rather with the h-polynomial

$$
h(\Delta, x)=h_{0}+h_{1} x+\cdots+h_{d} x^{d} .
$$

Sections 2-5 deal with combinatorial properties of simplicial subdivisions (or triangulations) $\Delta^{\prime}$ of a simplicial complex $\Delta$. The basic combinatorics of subdivisions depends in a subtle way on the precise notion of the term "subdivision." We will be concerned primarily with four types of subdivisions:

(a) General (or topological) subdivisions. Here we only require that (informally speaking) each simplex $2^{F}$ of the simplicial complex $\Delta$ is subdivided into a ball whose boundary is a subdivision of the boundary of $2^{F}$.

Received by the editors May 31, 1991.

1991 Mathematics Subject Classification. Primary 05E99; Secondary 06A07, 52B05.

Research carried out at M.I.T., Hebrew University (Jerusalem), the University of Augsburg, Tokai University (Hiratsuka), and the Royal Institute of Technology (Stockholm). Research at M.I.T. partially supported by NSF grant \#DMS-8401376, at Hebrew University by a grant from the U.S.-Israel Binational Science Foundation (BSF), and at Tokai University by the Matsumae International Foundation. 
(b) Geometric subdivisions. Here we want $\Delta$ and $\Delta^{\prime}$ to have geometric realizations (each face realized as a Euclidean simplex) such that $\Delta$ and $\Delta^{\prime}$ have the same underlying set, and such that every face of $\Delta^{\prime}$ is contained in a face of $\Delta$. This is the usual notion of subdivision used by topologists, e.g., [Mu, p. 83; Sp, p. 121].

(c) Quasi-geometric subdivisions. These subdivisions are topological subdivisions with one extra combinatorial condition; namely, no face of the subdivision $\Delta^{\prime}$ can have all its vertices on a face of $\Delta$ of smaller dimension. Clearly geometric subdivisions have this property, but quasi-geometric subdivisions are more general. As shown by Corollary 4.4, the defining property of quasi-geometric subdivisions is precisely what is needed to obtain the essential positivity result of Corollary 4.7. (See property (L3) of local $h$-vectors below.)

(d) Regular subdivisions. Loosely speaking, these are projections of a strictly convex polyhedral surface. They are an extensively studied subclass of the geometric subdivisions whose main significance for us is that we can apply the hard Lefschetz theorem for the decomposition theorem of intersection homology to obtain the unimodality result given by Theorem 5.2. (See property (L4) of local $h$-vectors below.)

The original motivation for $\S \S 2-5$ is the following question of Kalai and this author: If $\Delta^{\prime}$ is a (simplicial) subdivision of the Cohen-Macaulay complex $\Delta$, then is $h(\Delta) \leq h\left(\Delta^{\prime}\right)$ ? This question is answered affirmatively for quasi-geometric subdivisions, and remains open for topological subdivisions. The hypothesis that $\Delta$ is Cohen-Macaulay may even be relaxed somewhat; see Theorem 4.10. The key concept for the proof is that of the "local $h$-vector" $\ell_{V}(\Gamma)=\left(\ell_{0}, \ldots, \ell_{d}\right)$ or "local $h$-polynomial" $\ell_{V}(\Gamma, x)=\ell_{0}+\ell_{1} x+\cdots+\ell_{d} x^{d}$ of a subdivision $\Gamma$ of a $(d-1)$-simplex with vertex set $V$. The vector $\ell_{V}(\Gamma)$ has the following properties:

(L1) $\ell_{i}=\ell_{d-i}$ for all $i$.

(L2) For any pure simplicial complex $\Delta$ and any subdivision $\Delta^{\prime}$ of $\Delta$, we have

$$
h\left(\Delta^{\prime}, x\right)=\sum_{F \in \Delta} \ell_{F}\left(\Delta_{F}^{\prime}, x\right) h\left(\mathrm{lk}_{\Delta} F, x\right),
$$

where (i) $\Delta_{F}^{\prime}$ denotes the restriction of $\Delta^{\prime}$ to the face $F$ of $\Delta$, and (ii) $1 \mathrm{k}_{\Delta} F$ denotes the link of $F$ in $\Delta$.

(L3) For any quasi-geometric subdivision $\Gamma$ of a simplex $2^{V}, \ell_{V}(\Gamma) \geq 0$.

(L4) If $\Gamma$ is combinatorially equivalent to a regular subdivision of $2^{V}$ (as defined in Definition 5.1), then $\ell_{V}(\Gamma)$ is unimodal.

Of these properties, (L1) and (L2) have elementary proofs, (L3) uses machinery from commutative and homological algebra, while (L4) relies on deep results in intersection homology theory. Equation (2) shows exactly how the $h$ polynomial (or $h$-vector) of $\Delta^{\prime}$ depends on the properties of $\Delta$ alone (viz., the terms $h\left(\mathrm{lk}_{\Delta} F, x\right)$ ) and on how each face $F$ of $\Delta$ is subdivided (viz., $\left.\ell_{F}\left(\Delta_{F}^{\prime}, x\right)\right)$. It explains the terminology "local $h$-vector," since the local behav- 
ior of $\Delta$ at $F$ determines $h\left(\mathrm{k}_{\Delta} F, x\right)$, and thus $\ell_{F}\left(\Delta_{F}^{\prime}, x\right)$ is the contribution to $h\left(\Delta^{\prime}, x\right)$ of the subdivision $\Delta_{F}^{\prime}$ itself "at $F$."

If in (2) $\Delta$ is a Cohen-Macaulay simplicial complex and $\Delta^{\prime}$ is a quasigeometric subdivision of $\Delta$, then every term on the right-hand side is nonnegative. Moreover, when $F=\varnothing$ the corresponding term is $h(\Delta, x)$. Hence $h(\Delta, x) \leq h\left(\Delta^{\prime}, x\right)$, thus proving the above-mentioned conjecture of Kalai and this author in the quasi-geometric case.

Similarly, suppose we know that $\Delta$ is Cohen-Macaulay, and that for every $F \in \Delta$ the $h$-vector $h(\mathrm{lk} F)$ satisfies $h_{i}=h_{m-i}$ and $h_{0} \leq h_{1} \leq \cdots \leq$ $h_{\lfloor m / 2\rfloor}$, where $m=\# F$. (This is the case for the boundary complex of a simplicial $d$-polytope $\left[\mathrm{Sta}_{6}\right]$.) Let $g_{i}=h_{i}-h_{i-1}$, and define the $g$-vector $g(\Delta)=\left(g_{0}, g_{1}, \ldots, g_{\lfloor d / 2\rfloor}\right)$. Let $\Delta^{\prime}$ be a subdivision of $\Delta$ such that, for all $F \in \Delta$, the subdivision $\Delta_{F}^{\prime}$ of $F$ (or, more precisely, of the simplex $2^{F}$ ) is combinatorially equivalent to a regular subdivision. Then it is easy to deduce from (2) and (L4) that $g(\Delta) \leq g\left(\Delta^{\prime}\right)$. In other words, $g$-vectors (and not just $h$-vectors) of suitable simplicial complexes increase under regular subdivision. It is not known whether this fact remains true for more general subdivisions, such as geometric, quasi-geometric, or even topological.

A number of applications and examples related to our results on local $h$ vectors are presented in $\S \S 2-5$. For instance, in Proposition 3.4 we prove a conjecture of M. Kapranov concerning certain triangulations of simplices. In Problem 4.13-Example 4.17, we investigate the question of when local $h$ polynomials are 0 and relate it to the topic of "minimal triangulations." Proposition 4.20 deals with the interaction between the action of the symmetric group $\mathscr{S}_{d}$ on the first barycentric subdivision $\Gamma$ of a $(d-1)$-dimensional simplex $2^{V}$ (so $\Gamma$ is essentially the Coxeter complex of $\mathscr{S}_{d}$ ) and the local $h$-vector $\ell_{V}(\Gamma)$.

In $\S \S 6-9$ we extend the results of $\S \S 2-5$ to more general situations. For instance, we consider polyhedral subdivisions of polyhedral complexes. The $h$-vector is replaced with the "generalized $h$-vector" of [ $\left.\mathrm{Sta}_{10}\right]$. Properties (L1) and (L2) of the local $h$-vector carry over without difficulty, but we can only prove (L3) (as well as (L4)) in the special case when we can interpret the generalized $h$-vector in terms of the intersection homology of toric varieties.

Here is a brief description of some of the highlights of $\S \S 6-9$. In $\S 6$ we give a broad formal generalization, valid for any locally finite graded poset $P$, of the theory developed in $\S \S 2-5$ (the case where $P$ is a boolean algebra). For instance, Example 6.9 shows that Kazhdan-Lusztig polynomials can be incorporated within our theory (though we obtain no new results about these remarkable polynomials). In $\S 7$ we specialize the previous section to Eulerian posets, which have many special properties. In particular, in Definition 7.4 we give a vast formal generalization of the concept of subdivision of a simplicial complex. In Example 7.13 we discuss the connection between our methods and the theory of Ehrhart polynomials. In particular, an earlier result of Betke and McMullen [B-M, Theorem 1] is an Ehrhart polynomial analogue of (2) and provided some of the motivation for this paper. Section 8 is devoted to the effect on our theory 
of dualizing the poset $P$. As a consequence of our results we give (Corollary 8.8) a simple, conceptual proof of a conjecture of $\mathrm{G}$. Kalai, originally proved independently by Kalai and by A. Klapper. Finally in $\S 9$ we consider the possibility of a " $q$-analogue" of our theory of Eulerian posets. Unfortunately we show (Proposition 9.1) that a completely satisfactory $q$-analogue does not exist.

Throughout this paper we employ the following notation:

$$
\begin{aligned}
\mathbf{N} & =\{0,1,2, \ldots\}, \\
\mathbf{P} & =\{1,2,3, \ldots\}, \\
{[d] } & =\{1,2, \ldots, d\}, \quad \text { if } d \in \mathbf{P} .
\end{aligned}
$$

\section{THE LOCAL $h$-VECTOR OF A SUBDIVISION OF A SIMPLEX}

Let $V$ be a $d$-element vertex set, and let $2^{V}$ denote the simplex with vertex set $V$. (Thus $2^{V}$ is simply the set of all subsets of $V$.) Let $\Gamma$ denote a (finite) simplicial subdivision (or topological subdivision) of $2^{V}$. Formally, this means that $\Gamma$ is a simplicial complex and that we have a "subdivision map" $\sigma: \Gamma \rightarrow 2^{V}$ satisfying: (a) For every $W \subseteq V, \Gamma_{W}:=\sigma^{-1}\left(2^{W}\right)$ is a subcomplex of $\Gamma$ whose geometric realization $\left|\sigma^{-1}\left(2^{W}\right)\right|$ is homeomorphic to a ball of dimension $(\# W)-1$; and (b) $\sigma^{-1}(W)$ consists of the interior faces of the ball $\sigma^{-1}\left(2^{W}\right)$. (Hence $\sigma$ is surjective.) We call the subcomplex $\Gamma_{W}$ the restriction of $\Gamma$ to $W$ (or to $2^{W}$ ). If $F \in \Gamma_{W}$ then we say that $F$ lies on $W$. Hence for any $F \in \Gamma$, we have that $\sigma(F)$ is the unique smallest face of $2^{V}$ on which $F$ lies. We call this face $\sigma(F)$ the carrier of $F$. Thus we always have $\operatorname{dim} F \leq \operatorname{dim} \sigma(F)$.

For any simplicial complex $\Delta$ let $h(\Delta, x)$ denote its $h$-polynomial; i.e.,

$$
h(\Delta, x)=\sum_{i=0}^{d} h_{i} x^{i}
$$

where $h(\Delta)=\left(h_{0}, \ldots, h_{d}\right)$. The fundamental definition of this section is the following.

2.1. Definition. Let $\# V=d$. For any subdivision $\Gamma$ of a simplex $2^{V}$, define a polynomial $\ell_{V}(\Gamma, x)=\ell_{0}+\ell_{1} x+\cdots+\ell_{d} x^{d}$ by

$$
h(\Gamma, x)=\sum_{W \subseteq V} \ell_{W}\left(\Gamma_{W}, x\right) .
$$

We call $\ell_{V}(\Gamma, x)$ the local h-polynomial of $\Gamma$ (with respect to $2^{V}$ or to $V$ ), and we call $\ell_{V}(\Gamma):=\left(\ell_{0}, \ldots, \ell_{d}\right)$ the local $h$-vector of $\Gamma$ (with respect to $2^{V}$ or to $V)$. 
Note. Since equation (3) must be valid for all subdivisions of all simplices, in particular it holds with $\Gamma$ replaced by $\Gamma_{W}$. Thus by the Principle of InclusionExclusion (e.g., [ $\mathrm{Sta}_{11}$, Chapter 2.1]) we can invert (3) to obtain

$$
\ell_{V}(\Gamma, x)=\sum_{W \subseteq V}(-1)^{\#(V-W)} h\left(\Gamma_{W}, x\right) .
$$

Thus Definition 2.1 does indeed define $\ell_{V}(\Gamma, x)$. We could of course have made (4) the definition of $\ell_{V}(\Gamma, x)$, but we will see (following Corollary 7.7) that equation (3) generalizes more naturally than (4).

There is an alternative expression for $\ell_{V}(\Gamma, x)$ which is sometimes useful. Given $G \in \Gamma$, define the excess $e(G)$ of $G$ by the formula

$$
e(G)=\# \sigma(G)-\# G=\operatorname{dim} \sigma(G)-\operatorname{dim} G .
$$

\subsection{Proposition. We have}

$$
\ell_{V}(\Gamma, x)=\sum_{G \in \Gamma}(-1)^{d-\# G} x^{d-e(G)}(x-1)^{e(G)} .
$$

Proof. For any $(d-1)$-dimensional simplicial complex $\Delta$, it follows from (1) by substituting $1 / x$ for $x$ and multiplying by $x^{d}$ that

$$
h(\Delta, x)=\sum_{F \in \Delta} x^{\# F}(1-x)^{d-\# F} .
$$

Hence by (4),

$$
\begin{aligned}
\ell_{V}(\Gamma, x) & =\sum_{W \subseteq V}(-1)^{d-\# W} \sum_{G \in \Gamma_{W}} x^{\# G}(1-x)^{\# W-\# G} \\
& =\sum_{G \in \Gamma}(-1)^{d} x^{\# G}(1-x)^{-\# G} \sum_{W \supseteq \sigma(G)}(x-1)^{\# W} .
\end{aligned}
$$

By the binomial theorem, the inner sum is equal to $(x-1)^{\# \sigma(G)} x^{d-\# \sigma(G)}$, and the proof follows.

2.3. Example. (a) $\ell_{\varnothing}\left(2^{\varnothing}, x\right)=1$, where $\varnothing$ denotes the empty set.

(b) If $\Gamma=2^{V}$ (the trivial subdivision) where $\# V=d>0$, then $\ell_{V}\left(2^{V}, x\right)=$ 0 . This follows immediately from (3) and the fact that $h\left(2^{V}, x\right)=1$ for all $V$ (including $V=\varnothing$ ).

(c) If $\# V=2$ (so $\operatorname{dim} \Gamma=1$ ) and $\Gamma$ has $t$ interior vertices (so $t+2$ vertices in all), then $\ell_{V}(\Gamma, x)=t x$.

(d) If $\# V=3$ and $h(\Gamma, x)=h_{0}+h_{1} x+h_{2} x^{2}+h_{3} x^{3}$ (so $h_{0}=1$ and $\left.h_{3}=0\right)$, then

$$
\ell_{V}(\Gamma, x)=h_{2} x+h_{2} x^{2}
$$

Moreover (see (f) below), $h_{2}$ is equal to the number of interior vertices of $\Gamma$.

(e) From the above examples it follows that $\ell_{V}(\Gamma, x)$ depends only on $\Gamma$ as an abstract simplicial complex (in fact, only on $h(\Gamma, x)$ ) when $\# V \leq 3$. This 
fact is no longer true for $\# V \geq 4$. For instance, let $\# V=4$. Let $\Gamma_{1}$ be the stellar subdivision of $2^{V}$ obtained by adding a vertex to the center of a 2 -face and 3-face. (Of course the only 3-face is $V$ itself.) Similarly obtain $\Gamma_{2}$ by adding a vertex to the center of a 1 -face and 3-face. Then $\Gamma_{1}$ and $\Gamma_{2}$ are isomorphic as abstract simplicial complexes (with $h\left(\Gamma_{1}, x\right)=h\left(\Gamma_{2}, x\right)=1+$ $\left.2 x+2 x^{2}+x^{3}\right)$, but $\ell_{V}\left(\Gamma_{1}, x\right)=x+x^{2}+x^{3}$ and $\ell_{V}\left(\Gamma_{2}, x\right)=x+2 x^{2}+x^{3}$.

(f) Since $h_{d}(\Delta)=0$ for any triangulation $\Delta$ of a $(d-1)$-ball, the only term on the right-hand side of (4) for which the coefficient of $x^{d-1}$ can be nonzero is given by $W=V$. Moreover, the coefficient of $x^{d}$ is always 0 unless $\Gamma=2^{\varnothing}=\{\varnothing\}$. Hence if $\ell_{V}(\Gamma, x)=\sum_{0}^{d} \ell_{i} x^{i}$, then $\ell_{d}=0$ unless $d=0$, and $\ell_{d-1}=h_{d-1}(\Gamma)$. Let us note that, e.g., from [ $\mathrm{Ma}_{1}$, Proposition 1.1, $\left.\operatorname{Sta}_{1},(1.1)\right]$ we have that $h_{d-1}(\Gamma)$ is the number of interior vertices of $\Gamma$ (i.e., the number of vertices whose carrier is $V$ ). From Theorem 3.3 below we also see that $\ell_{1}=h_{d-1}(\Gamma)$ and $\ell_{0}=0$ unless $d=0$. (It is also not difficult to obtain the values of $\ell_{0}$ and $\ell_{1}$ directly from (3) or (4).) Thus $\ell_{0}, \ell_{1}, \ell_{d-1}$, and $\ell_{d}$ depend only on $h(\Gamma)$, while (e) above shows that $\ell_{2}$ cannot be computed from just the structure of $\Gamma$ as an abstract simplicial complex. It is easy to extend this example to show that any $\ell_{i}$ with $2 \leq i \leq d-2$ does not depend only on $\Gamma$ as an abstract simplicial complex.

(g) Assume $V \neq \varnothing$ and consider Proposition 2.2. No faces $G \in \Gamma$ satisfy $e(G)=d$, while $e(G)=d-1$ if and only if $G$ is an interior vertex of $\Gamma$. Taking the coefficient of $x$ yields that $\ell_{1}$ is the number of interior vertices, as obtained in (f) above. Similarly, $e(G)=d-2$ if and only if either (i) $G$ is an interior edge, or (ii) $G$ is a vertex whose carrier is a $(d-2)$-face of $2^{V}$. Let $f_{i}^{\circ}$ denote the number of interior $i$-faces of $\Gamma$, and let $\tilde{f}_{0}$ be the number of vertices $v$ of $\Gamma$ for which $\# \sigma(v)=d-1$. Then taking the coefficient of $x^{2}$ in (5) yields

$$
\ell_{2}=-(d-1) f_{0}^{\circ}+f_{1}^{\circ}-\tilde{f}_{0} .
$$

By Theorem 3.3, this is also the value of $\ell_{d-2}$. Of course similar formulas hold for any $\ell_{i}$, but in general they seem too complex to afford much insight.

(h) (C. Chan) Let $V=\{1,2,3,4\}$. Let $\Gamma$ be the subdivision of $2^{V}$ obtained by adding a vertex 5 inside face 123 , and letting the facets of $\Gamma$ be 1234 and 1235. (Note that the interior of a "curved triangle" 123 has been inserted into the interior of the simplex 1234.) Then $\ell(\Gamma, x)=-x^{2}$. In $\S 4$ we will see what bad property of $\Gamma$ causes the local $h$-vector to have a negative entry.

(i) Let $\# V=d$. A natural subdivision of $2^{V}$ is the (first) barycentric subdivision $\Gamma=\operatorname{sd}\left(2^{V}\right)$. $\Gamma$ may also be regarded as the Coxeter complex of the symmetric group $\mathscr{S}_{d}$. It is well known that

$$
h(\Gamma, x)=\sum_{i=0}^{d-1} A_{d, i+1} x^{i}
$$


where $A_{d, i+1}$ denotes the Eulerian number [C, Chapter 6.5, $\mathrm{Sta}_{11}$, p. 22]. (Thus $x h(\Gamma, x)$ is the Eulerian polynomial $A_{d}(x)$.) The Eulerian numbers have the following combinatorial interpretation (among others) [ $\mathrm{Sta}_{11}$, Proposition 1.3.12]:

$$
h(\Gamma, x)=\sum_{w \in \mathscr{S}_{d}} x^{\mathrm{ex}(w)},
$$

where $\operatorname{ex}(w)$ denotes the number of excedances of $w$, defined by

$$
\operatorname{ex}(w)=\#\{i: w(i)>i\} .
$$

We now give a similar interpretation of $\ell_{V}(\Gamma, x)$.

2.4. Proposition. With notation as above, we have

$$
\ell_{V}(\Gamma, x)=\sum_{w \in \mathscr{D}_{d}} x^{e x(w)},
$$

where $\mathscr{D}_{d}$ denotes the set of all derangements (i.e., $w(i) \neq i$ for all $\left.i\right)$ in $\mathscr{S}_{d}$. Proof. Let us write $h^{j}(x)$ for $h(\Gamma, x)$ and $\ell^{j}(x)$ for $\ell_{Y}(\Gamma, x)$ when $\# Y=j$ and $\Gamma=\operatorname{sd}\left(2^{Y}\right)$. Since for any $W \subseteq V$ we have $\Gamma_{W}=\operatorname{sd}\left(2^{W}\right)$, and since $V$ has $\left(\begin{array}{l}d \\ i\end{array}\right)$ faces of cardinality $i$, it follows from (3) that

$$
h^{d}(x)=\sum_{i=0}^{d}\left(\begin{array}{l}
d \\
i
\end{array}\right) \ell^{i}(x) .
$$

Hence by (7) we need to show

$$
\sum_{w \in \mathscr{S}_{d}} x^{\mathrm{ex}(w)}=\sum_{i=0}^{d}\left(\begin{array}{l}
d \\
i
\end{array}\right) \sum_{v \in \mathscr{D}_{i}} x^{\mathrm{ex}(v)} .
$$

But if $w \in \mathscr{S}_{d}$ has a given set $S$ of $d-i$ fixed points, then the remaining set $T=[d]-S$ of $i$ elements which $w$ permutes can form any derangement of $T$, while relabeling the elements of $T$ by $1, \ldots, i$ in increasing order preserves the number of excedances. Since there are $\left(\begin{array}{c}d \\ d-i\end{array}\right)=\left(\begin{array}{c}d \\ i\end{array}\right)$ choices for $S$, (8) follows.

For further information about excedances, see $\left[\mathrm{Br}_{2}\right]$. For a refinement of Proposition 2.4, see Proposition 4.20 below.

\section{ELEMENTARY PROPERTIES OF LOCAL $h$-VECTORS}

We now come to the basic properties of local $h$-vectors. We have four results in all (Theorem 3.2, Theorem 3.3, Corollary 4.7, and Theorem 5.2). The first of these results clarifies the terminology "local $h$-vector." 
3.1. Lemma. Let $\Gamma$ be a pure $(d-1)$-dimensional simplicial complex. ("Pure" means that all maximal faces have $d$ elements.) Then

$$
\sum_{F \in \Gamma}(-1)^{d-\# F} h\left(\mathrm{lk}_{\Gamma} F, x\right)=-(x-1)^{d} \tilde{\chi}(\Gamma),
$$

where $\tilde{\chi}(\Gamma)=-1+f_{0}-f_{1}+\cdots$, the reduced Euler characteristic of $\Gamma$.

Proof. Since $\Gamma$ is pure we have $\operatorname{dim}\left(\mathrm{k}_{\Gamma} F\right)=d-\# F-1$ for all $F \in \Gamma$. Thus by (6) we have

$$
\begin{aligned}
\sum_{F \in \Gamma}(-1)^{d-\# F} h\left(\mathrm{k}_{\Gamma} F, x\right) & =\sum_{F \in \Gamma}(-1)^{d-\# F} \sum_{F \subseteq G \in \Gamma} x^{\#(G-F)}(1-x)^{d-\# G} \\
& =\sum_{G \in \Gamma}(-1)^{d} x^{\# G}(1-x)^{d-\# G} \sum_{F \subseteq G}(-x)^{-\# F} \\
& =\sum_{G \in \Gamma}(-1)^{d} x^{\# G}(1-x)^{d-\# G}\left(1-\frac{1}{x}\right)^{\# G} \\
& =(x-1)^{d} \sum_{G \in \Gamma}(-1)^{\# G} \\
& =-(x-1)^{d} \tilde{\chi}(\Gamma) .
\end{aligned}
$$

3.2. Theorem. Let $\Delta$ be a pure $(d-1)$-dimensional simplicial complex, and let $\Delta^{\prime}$ be a simplicial subdivision of $\Delta$. Then

$$
h\left(\Delta^{\prime}, x\right)=\sum_{F \in \Delta} \ell_{F}\left(\Delta_{F}^{\prime}, x\right) h\left(\mathrm{lk}_{\Delta} F, x\right) .
$$

Proof. Let $R$ denote the right-hand side of (9). By (4) and the previous lemma, we have

$$
\begin{aligned}
R & =\sum_{F \in \Delta}\left[\sum_{G \subseteq F}(-1)^{\#(F-G)} h\left(\Delta_{G}^{\prime}, x\right)\right] h\left(\mathrm{lk}_{\Delta} F, x\right) \\
& =\sum_{G \in \Delta} h\left(\Delta_{G}^{\prime}, x\right)\left[\sum_{F \supseteq G}(-1)^{\#(F-G)} h\left(\mathrm{lk}_{\Delta} F, x\right)\right] \\
& =-\sum_{G \in \Delta} h\left(\Delta_{G}^{\prime}, x\right)(1-x)^{d-\# G} \tilde{\chi}\left(\mathrm{lk}_{\Delta} G\right) .
\end{aligned}
$$

Now using (6) we get

$$
\begin{aligned}
R & =-\sum_{G \in \Delta}\left[\sum_{H \in \Delta_{G}^{\prime}} x^{\# H}(1-x)^{\#(G-H)}\right](1-x)^{d-\# G} \tilde{\chi}\left(\mathrm{lk}_{\Delta} G\right) \\
& =-\sum_{H \in \Delta^{\prime}} x^{\# H}(1-x)^{d-\# H} \sum_{G \supseteq \sigma(H)} \tilde{\chi}\left(\mathrm{lk}_{\Delta} G\right) .
\end{aligned}
$$

Let $P$ be the poset of faces of $\Delta$ (ordered by inclusion), with a maximal element $\hat{1}$ adjoined. Let $\mu$ denote the Möbius function of $P\left[\mathrm{Sta}_{11}\right.$, Chapter 
3.7]. By [ $\mathrm{Sta}_{11}$, Proposition 3.8.8] we have

$$
\tilde{\chi}\left(\mathrm{lk}_{\Delta} G\right)=\mu(G, \hat{1}) .
$$

Hence by the fundamental recurrence [ $\operatorname{Sta}_{11}$, Chapter $\left.3.7,(14)\right]$ for $\mu$, we have

$$
\sum_{G \supseteq \sigma(H)} \tilde{\chi}\left(\mathrm{lk}_{\Delta} G\right)=-\mu(\hat{1}, \hat{1})=-1 .
$$

Therefore

$$
R=\sum_{H \in \Delta^{\prime}} x^{\# H}(1-x)^{d-\# H}=h\left(\Delta^{\prime}, x\right),
$$

by (6).

For a generalization of Theorem 3.2, see Theorem 7.8 (for which we give a slicker but somewhat less elementary proof).

Our second main result on local $h$-vectors is a "self-duality" theorem analogous to the Dehn-Sommerville equations.

3.3. Theorem. Let $\Gamma$ be a (finite) simplicial subdivision of the simplex $2^{V}$, where $\# V=d$. Then

$$
x^{d} \ell_{V}(\Gamma, 1 / x)=\ell_{V}(\Gamma, x) .
$$

Equivalently, $\ell_{i}=\ell_{d-i}$ for all $i$.

Proof. A more general result will be proved later (Corollary 7.7).

As an application of Theorem 3.3, we give a proof of an unpublished conjecture of M. Kapranov related to the work of [G-Z-K].

3.4. Proposition. Let $\Gamma$ be a simplicial subdivision (or triangulation) of the simplex $2^{V}$ (where \#V=d) such that every vertex $v$ of $\Gamma$ has excess (as defined in $\S 2) e(v)=0$ or 1 . It is then easily seen that every face $G \in \Gamma$ satisfies $e(G) \leq \# G$. Let $\alpha(\Gamma)$ be the number of faces $G$ of $\Gamma$ satisfying $e(G)=\# G$ (including $G=\varnothing$, for which $e(\varnothing)=0=\# \varnothing$ ). Then $\alpha(\Gamma)=f_{d-1}(\Gamma)$, the number of facets of $\Gamma$.

Proof. Let $\varnothing \neq W \subseteq V$, with $\# W=s$. According to Proposition 2.2, we have

$$
\ell_{W}\left(\Gamma_{W}, x\right)=\sum_{G \in \Gamma_{W}}(-1)^{s-\# G} x^{s-e(G)}(x-1)^{e(G)} .
$$

If $\# G=r$ then as mentioned above $e(G) \leq r$, while since $G \in \Gamma_{W}$ with $\Gamma_{W}=s$ we have $e(G) \leq s-r$. Hence $2 e(G) \leq s$. Suppose $s$ is odd, say $s=2 t+1$. Then $s-e(G) \geq t+1$, so $\ell_{W}\left(\Gamma_{W}, x\right)$ is divisible by $x^{t+1}$. Since $\ell_{W}\left(\Gamma_{W}, x\right)=x^{s} \ell_{W}\left(\Gamma_{W}, 1 / x\right)$ by Theorem 3.3 , it follows that $\ell_{W}\left(\Gamma_{W}, x\right)=0$. Now suppose $s$ is even, say $s=2 t$. We then get $s-e(G) \geq t$, so $\ell_{W}\left(\Gamma_{W}, x\right)$ is divisible by $x^{t}$. Again by Theorem 3.3 we have $\ell_{W}\left(\Gamma_{W}, x\right)=c_{W} x^{t}$ for some integer $c_{W}$. To obtain the value of $c_{W}$, note that from the above expression 
for $\ell_{W}\left(\Gamma_{W}, x\right)$ we have that the coefficient of $x^{t}$ in $\ell_{W}\left(\Gamma_{W}, x\right)$ is given by

$$
c_{W}=\sum_{\substack{G \in \Gamma_{W} \\ e(G)=t}}(-1)^{s-\# G}(-1)^{e(G)} .
$$

The only way we can have $G \in \Gamma_{W}$ and $e(G)=t$ is for $\# G=t$ and $\sigma(G)=W$. Hence $c_{W}$ is just the number of faces $G$ of $\Gamma_{W}$ for which $\sigma(G)=W$ and $e(G)=\# G$. Setting $x=1$ in (3) yields that $h(\Gamma, 1)$ is the number of faces $G$ of $\Gamma$ for which $e(G)=\# G$. Since $h(\Gamma, 1)=f_{d-1}(\Gamma)$, the proof follows.

\section{NONNEGATIVITY OF LOCAL $h$-VECTORS}

Our aim in this section is to establish nonnegativity of the local $h$-vector $\ell_{V}(\Gamma)$ for certain subdivisions $\Gamma$ of $2^{V}$. We begin by defining the subdivisions of interest to us. First define a geometric simplex to be the convex hull of a set of affinely independent points in $\mathbf{R}^{n}$.

4.1. Definition. (a) A simplicial subdivision $\Delta^{\prime}$ of a simplicial complex $\Delta$ is quasi-geometric if for every face $F$ of $\Delta^{\prime}$, there does not exist a face $W$ of $\Delta$ such that (i) $\operatorname{dim} W<\operatorname{dim} F$, and (ii) for every vertex $v$ of $F, v$ lies on some subset $Y$ (depending on $v$ ) of $W$. Equivalently, no face of $\Delta^{\prime}$ has all its vertices on the closure of a face of $\Delta$ of lower dimension.

(b) A simplicial subdivision $\Gamma$ of a simplex $2^{V}$ is geometric if it can be realized in $\mathbf{R}^{n}$ as a subdivision of a geometric simplex into geometric simplices.

Clearly every geometric subdivision $\Gamma$ of $2^{V}$ is quasi-geometric. Example 2.3(h) gives a subdivision $\Gamma$ of $2^{V}$ for $V=\{1,2,3,4\}$ which is not quasigeometric. A similar example can be given for $V=\{1,2,3\}$; viz., add a vertex 4 inside the face 12 of $2^{V}$, and let the facets of $\Gamma$ be 123 and 124 . On the other hand, it is known that there exist quasi-geometric subdivisions of simplices which are not geometric. For instance, take a non-PL 5-sphere $\Gamma^{\prime}$ (existence guaranteed by work of Edwards; see [Dav]), and remove any facet $V=\left\{x_{1}, \ldots, x_{6}\right\}$ "away from" vertices whose links are not spheres. The remaining complex $\Gamma=\Gamma^{\prime}-\{V\}$ is a quasi-geometric but nongeometric subdivision of the simplex $2^{V}$. There also exist PL examples, e.g., quasi-geometric subdivisions of a 3-simplex for which the boundary of some 2-face is knotted.

The proofs of Theorem 3.2 and 3.3 (the latter yet to be given) are purely formal, but for our next result we do not see how to avoid introducing machinery from commutative and homological algebra. Given a simplicial subdivision $\Gamma$ of $2^{V}$, where $V=\left\{x_{1}, \ldots, x_{d}\right\}$, let $K[\Gamma]$ denote the face ring (or StanleyReisner ring) of $\Gamma$ over the field $K$, as defined e.g. in [ $\mathrm{Sta}_{8}$, p. 62].

4.2. Definition. A homogeneous system of parameters (h.s.o.p.) $\theta_{1}, \ldots, \theta_{d}$ of degree one of $K[\Gamma]$ is called special if each $\theta_{i}$ is a linear combination of vertices $x$ of $\Gamma$ which do not lie on the closure $2^{V-\left\{x_{i}\right\}}$ of the face $V-\left\{x_{i}\right\}$ of $2^{V}$. 
We want to show that a special h.s.o.p. always exists when $\Gamma$ is quasigeometric (and when $K$ is "large"). To do this we use the criterion [ $\operatorname{Sta}_{5}$, Remark on p. 150; Bi, p. 66] for the existence of an h.s.o.p. of degree one. Since this result is stated in $\left[\mathrm{Sta}_{5} ; \mathrm{Bi}\right]$ without proof and is only stated and proved implicitly in [K-K], for the sake of completeness we will sketch a proof.

4.3. Proposition. Let $\Delta$ be a $(d-1)$-dimensional simplicial complex. Let $\theta_{1}, \ldots, \theta_{d} \in K[\Delta]_{1}$ (the space of elements of $K[\Delta]$ which are homogeneous of degree one). Then $\theta_{1}, \ldots, \theta_{d}$ is an h.s.o.p. if and only if for every face $F$ of $\Delta$ (or just for every maximal face), if we set in $\theta_{1}, \ldots, \theta_{d}$ each vertex $x_{i} \notin F$ equal to zero (in other words, if we restrict each $\theta_{i}$ to $F$ ), then $\theta_{1}, \ldots, \theta_{d}$ span the vector space with basis $F$.

Proof (sketch). The "only if" part is easy. To prove the "if" part, let $R=$ $K[\Delta] /\left(\theta_{1}, \ldots, \theta_{d}\right)$. Assuming the conditions on the $\theta$ 's given in the statement of the proposition, we want to show $\operatorname{dim}_{K} R<\infty$. Let $R_{+}=R_{1} \oplus R_{2} \oplus \cdots$. If $F$ is a maximal face of $\Delta$, then one easily sees that $R_{+}$annihilates in $R$ the monomial $x^{F}:=\prod_{x \in F} x$. Thus $\operatorname{dim}_{K} R<\infty$ if and only if

$$
\operatorname{dim}_{K} R /\left(x^{F}: F \text { is a maximal face of } \Delta\right)<\infty .
$$

Now repeat the argument for $\Delta^{\prime}=\Delta-\{$ maximal faces of $\Delta\}$, etc., until we reach $R /\left(x^{F}: F\right.$ is any face of $\left.\Delta\right)=0$, which is clearly finite-dimensional over $K$.

4.4. Corollary. Let $\Gamma$ be a simplicial subdivision of $2^{V}$, where $\# V=d$, and let $K$ be an infinite field. Then there exists a special h.s.o.p. $\theta_{1}, \ldots, \theta_{d}$ for $K[\Gamma]$ if and only if $\Gamma$ is quasi-geometric.

Proof. Assume $\Gamma$ is quasi-geometric. Choose $\theta_{1}, \ldots, \theta_{d} \in K[\Gamma]_{1}$ as "generically" as possible subject to satisfying the condition for being special, so $\theta_{i}$ is a "generic" linear combination of vertices $x$ of $\Gamma$ which do not lie on the closure of the face $V-\left\{x_{i}\right\}$ of $2^{V}$. Let $F=\left\{y_{1}, \ldots, y_{d}\right\}$ be a facet of $\Gamma$. By the previous proposition, we need to show that, when $\theta_{1}, \ldots, \theta_{d}$ are restricted to $F$, they remain linearly independent. Since $\Gamma$ is quasi-geometric, we can assume by a simple application of the Marriage Theorem that the $y_{i}$ 's are labelled so that $y_{i}$ does not lie on the closure of the face $V-\left\{x_{i}\right\}$. Thus the coefficient of $y_{i}$ in $\theta_{i}$ is nonzero. Since $y_{1}, \ldots, y_{d}$ are linearly independent and the nonzero coefficients of $\theta_{1}, \ldots, \theta_{d}$ are generic, it follows that $\theta_{1}, \ldots, \theta_{d}$ restricted to $F$ are linearly independent.

For the converse, assume $\Gamma$ is not quasi-geometric. Let $F$ be a face of $\Gamma$, say of dimension $e-1$, whose vertices lie on the closure of some face of $2^{V}$ of dimension at most $e-2$. If $\theta_{1}, \ldots, \theta_{d}$ were a special h.s.o.p. then at most $e-2$ of the $\theta_{i}$ 's would involve any of the vertices of $F$, violating Proposition 4.3 .

Now let (int $\Gamma$ ) denote the ideal of $K[\Gamma]$ generated by the interior faces of $\Gamma$; i.e., (int $\Gamma)=\left(x^{F}: \sigma(F)=V\right)$. (We have by $\left[\mathrm{Sta}_{8}\right.$, Theorem 7.3] that 
(int $\Gamma$ ) is the canonical module of $K[\Gamma]$, but we do not need this fact here except for Corollary 4.19 , which is unrelated to the rest of the paper.)

4.5. Definition. Let $\Gamma$ be a quasi-geometric simplicial subdivision of $2^{V}$, where $\# V=d$. Let $\theta_{1}, \ldots, \theta_{d}$ be a special h.s.o.p. for $K[\Gamma]$. Then we denote the image of (int $\Gamma$ ) in $R_{\Gamma}:=K[\Gamma] /\left(\theta_{1}, \ldots, \theta_{d}\right)$ by $L_{V}(\Gamma)$ and call it the local face module of $\Gamma$ (with respect to $V$ and $\theta_{1}, \ldots, \theta_{d}$ ).

The module $L_{V}(\Gamma)$ is a graded ideal of $R_{\Gamma}$. Since $\Gamma$ is a triangulation of a ball it is Cohen-Macaulay (see [ $\mathrm{Sta}_{8}$, Chapter II, Corollary 4.2]), and therefore by standard properties of face rings (e.g., [ $\left.\left.\mathrm{Sta}_{9}\right]\right)$, the Hilbert function of $R_{\Gamma}=$ $R_{0} \oplus R_{1} \oplus \cdots$ is given by

$$
H\left(R_{\Gamma}, i\right):=\operatorname{dim}_{K} R_{i}=h_{i}(\Gamma) .
$$

Hence (since $h_{i}=0$ for $i>d$ ) the grading of $L_{V}(\Gamma)$ has the form

$$
L_{V}(\Gamma)=L_{0} \oplus \cdots \oplus L_{d},
$$

where $\operatorname{dim}_{K} L_{i} \leq h_{i}(\Gamma)$. Our main result on the ideal $L_{V}(\Gamma)$ is the following. 4.6. Theorem. The Hilbert function of $L_{V}(\Gamma)$ is given by

$$
\operatorname{dim}_{K} L_{i}=\ell_{i}
$$

where $\ell_{V}(\Gamma)=\left(\ell_{0}, \ldots, \ell_{d}\right)$ is the local h-vector of $\Gamma$.

4.7. Corollary. If $\Gamma$ is quasi-geometric, then the local h-vector $\ell_{V}(\Gamma)$ satisfies $\ell_{i} \geq 0$.

4.8. Corollary. If $\Gamma$ is quasi-geometric, then the vector

$$
h(\Gamma)-\ell_{V}(\Gamma)=\left(h_{0}-\ell_{0}, \ldots, h_{d}-\ell_{d}\right)
$$

is an O-sequence (also called an $M$-vector), as defined in $\left[\mathrm{Sta}_{3}, \mathrm{p} .60\right]$.

Proof of Corollary 4.8. The Hilbert function of $R_{\Gamma} / L_{V}(\Gamma)$ is given by

$$
H\left(R_{\Gamma} / L_{V}(\Gamma), i\right)=h_{i}-\ell_{i}
$$

The proof follows from the characterization of Hilbert functions in terms of $O$-sequences [ $\mathrm{Sta}_{3}$, Theorem 2.2].

Note. Before proving Theorem 4.6, let us note that the assumption that the h.s.o.p. $\theta_{1}, \ldots, \theta_{d} \in K[\Gamma]_{1}$ is special is essential (still assuming that $\Gamma$ is quasi-geometric). If for instance $\partial \Gamma$ is the boundary complex of a simplicial polytope and $\theta_{1}, \ldots, \theta_{d} \in K[\Gamma]_{1}$ is generic, then it can be deduced from [ Sta $_{6}$ ] that the Hilbert function $H(i)=H\left((\right.$ int $\left.\Gamma) /\left(\theta_{1}, \ldots, \theta_{d}\right), i\right)$ of the image of (int $\Gamma)$ in $K[\Gamma] /\left(\theta_{1}, \ldots, \theta_{d}\right)$ is given by

$$
H(i)= \begin{cases}h_{d-i}(\Gamma), & 0 \leq i \leq\lfloor d / 2\rfloor, \\ h_{i}(\Gamma), & 1+\lfloor d / 2\rfloor \leq i \leq d .\end{cases}
$$

Only in rare cases do we have $H(i)=\ell_{i}$. Let us also note that it follows from the fact that $H\left(K[\Gamma] /\left(\theta_{1}, \ldots, \theta_{d}\right), i\right)=h_{i}(\Gamma)$ for any h.s.o.p. $\theta_{1}, \ldots, \theta_{d} \in$ 
$K[\Gamma]_{1}$ (since $K[\Gamma]$ is Cohen-Macaulay) and from Proposition 4.18 below that the function $H(i)$ of $(10)$ is the largest possible Hilbert function of any image of $K[$ int $\Gamma]$ in a ring of the form $K[\Gamma] /\left(\theta_{1}, \ldots, \theta_{d}\right)$, where $\theta_{1}, \ldots, \theta_{d} \in K[\Gamma]_{1}$ is an h.s.o.p. It might be interesting to consider what the smallest such Hilbert function can be. When $\Gamma$ is quasi-geometric it is not always achieved by the ideal $L_{V}[\Gamma]$, since by Example 2.3(e) a different choice of $V$ may produce a smaller Hilbert function.

The proof of Theorem 4.6 rests upon a homological lemma. For any module $M$ over a (commutative) ring $A$ and submodules $N_{1}, \ldots, N_{d}$, there is a natural complex of $A$-modules

$$
M \rightarrow \coprod_{i} M / N_{i} \rightarrow \coprod_{i<j} M /\left(N_{i}+N_{j}\right) \rightarrow \cdots \rightarrow M /\left(N_{1}+\cdots+N_{d}\right) \rightarrow 0,
$$

whose maps are the usual coboundary maps.

4.9. Lemma. Let $\Gamma$ be a quasi-geometric simplicial subdivision of $2^{V}$, where $V=\left\{x_{1}, \ldots, x_{d}\right\}$. In equation (11), choose $M=K[\Gamma]$ (regarded as a module over itself), and let $N_{i}$ be the ideal of $K[\Gamma]$ generated by all monomials $x^{F}$ for which $F \in \Gamma$ does not lie on the closure of the face $V-\left\{x_{i}\right\}$ of $2^{V}$ (i.e., $x_{i} \in \sigma(G)$ for some $\left.G \subseteq F\right)$. Thus if $S=\left\{x_{i_{1}}, \ldots, x_{i_{r}}\right\} \subseteq V$ and $T=V-S$, then

$$
K[\Gamma] /\left(N_{i_{1}}+\cdots+N_{i_{r}}\right) \cong K\left[\Gamma_{T}\right]
$$

where $\Gamma_{T}$ denotes the restriction of $\Gamma$ to $T$. Denote the resulting complex (11) by $\mathscr{K}=\mathscr{K}(\Gamma)$. Let $\theta=\left\{\theta_{1}, \ldots, \theta_{d}\right\}$ be a special h.s.o.p. for $K[\Gamma]$. Let $\mathscr{K} / \theta \mathscr{K}$ denote the complex obtained from $\mathscr{K}$ by modding out by $\theta_{1}, \ldots, \theta_{d}$, or equivalently, by tensoring $\mathscr{K}$ with $K[\Gamma] / \theta K[\Gamma]$. Then $\mathscr{K} / \theta \mathscr{K}$ is exact.

Proof. We prove by induction on $r$ that $\mathscr{K} /\left(\theta_{1}, \ldots, \theta_{r}\right) \mathscr{K}$ is exact. For $r=0$, we need to prove that $\mathscr{K}$ is exact. To see this, note that the modules appearing in (11) are multigraded by $\mathbf{N}^{m}$, where $m$ is the number of vertices of $\Gamma$ and $\mathbf{N}=\{0,1, \ldots\}$. The grading is given by $\operatorname{deg} x_{1}^{\alpha_{1}} \cdots x_{m}^{\alpha_{m}}=\left(\alpha_{1}, \ldots, \alpha_{m}\right)$. (This is the "fine grading" of $\left[\mathrm{Sta}_{8}\right.$, p. 63].) The maps in (11) preserve degree, so it suffices to show that the restriction $\mathscr{K}_{\alpha}$ of $\mathscr{K}$ to its part of degree $\alpha \in \mathbf{N}^{m}$ is exact. If $\alpha=\left(\alpha_{1}, \ldots, \alpha_{m}\right)$, then $\mathscr{K}_{\alpha}^{\alpha}$ will be the zero complex unless $\left\{x_{j}: \alpha_{j} \neq 0\right\}$ is a face $F=F_{\alpha}$ of $\Gamma$. In this case suppose that the carrier $\sigma(F)$ of $F$ satisfies $\# \sigma(F)=i$. Then $\mathscr{K}_{\alpha}$ may be identified with the usual augmented cochain complex (followed by some irrelevant zero terms), with the first term (which is 0 ) omitted, of a $(d-i-1)$-simplex $\Sigma$. If $i<d$ then $\Sigma$ and hence $\mathscr{K}_{\alpha}$ is acyclic When $i=d$ we obtain the complex

$$
K \rightarrow 0 \rightarrow 0 \rightarrow \cdots \rightarrow 0
$$

which is also acyclic. Hence $\mathscr{K}$ is exact as claimed.

Now assume the hypothesis for $r$. Proofs of the induction step follow from arguments shown to me by $\mathrm{K}$. Watanabe (based on spectral sequences), 
S. Yuzvinsky (based on sheaf cohomology), and M. Hochster (also based on spectral sequences). Hochster then reformulated his argument in more elementary terms. It is this argument of Hochster on which the proof given here is based.

Let an overhead bar denote the modding out by $\left\{\theta_{1}, \ldots, \theta_{r}\right\}$, so by assumption the complex

$$
\overline{K[\Gamma]} \rightarrow \coprod_{i} \overline{K[\Gamma]} / \bar{N}_{i} \rightarrow \cdots \rightarrow 0
$$

is exact. Let $C^{i}$ denote the $i$ th term of the above complex, beginning with $C^{0}=\overline{K[\Gamma]}$, so we have a complex $\mathscr{C}$ given by

$$
\mathscr{C}: 0 \rightarrow C^{0} \rightarrow C^{1} \rightarrow \cdots \rightarrow C^{d} \rightarrow 0
$$

which is exact except (possibly) at $C^{0}$. Form the exact sequence $0 \rightarrow \mathscr{B} \rightarrow$ $\mathscr{C} \stackrel{\theta_{r+1}}{\rightarrow} \mathscr{C} \rightarrow \mathscr{D} \rightarrow 0$ of complexes, where the map $\mathscr{C} \rightarrow \mathscr{C}$ is given by multiplication by $\theta_{r+1}$, and where $\mathscr{B}=\operatorname{ker}(\mathscr{C} \rightarrow \mathscr{C}), \mathscr{D}=\operatorname{coker}(\mathscr{C} \rightarrow \mathscr{C})=\mathscr{C} / \theta_{r+1} \mathscr{C}$. In more expanded notation we have

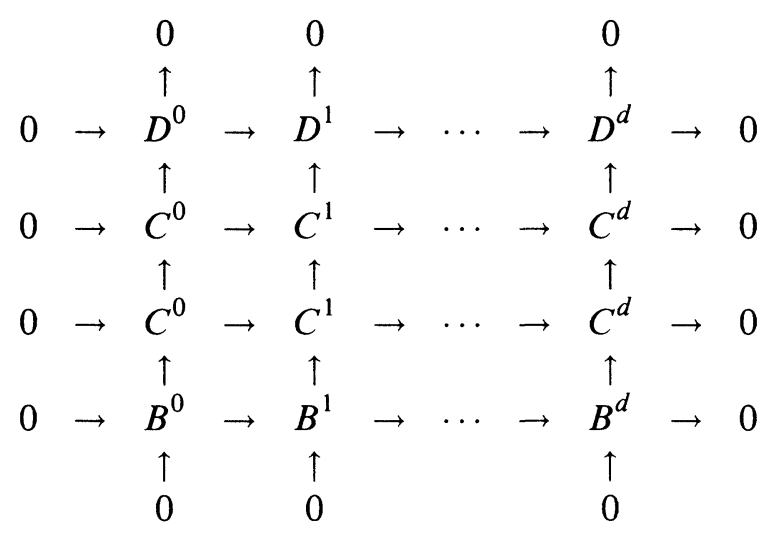

We want to show that $\mathscr{D}$ is exact except at $D^{0}$. Consider

$$
C^{j}=\coprod_{i_{1}<\cdots<i_{j}} \overline{K[\Gamma]} /\left(\bar{N}_{i_{1}}+\cdots+\bar{N}_{i_{j}}\right) .
$$

Since $\theta_{1}, \ldots, \theta_{d}$ is special, $\theta_{r+1}$ will annihilate the term

$$
\overline{K[\Gamma]} /\left(\bar{N}_{i_{1}}+\cdots+\bar{N}_{i_{j}}\right) \cong \overline{K\left[\Gamma_{T}\right]}
$$

of $C^{j}$ if some $i_{s}=r+1$, while $\theta_{r+1}$ will be a nonzero-divisor on this term otherwise. Hence the kernel $B^{j}$ of the map $C^{j \stackrel{\theta_{r+1}}{\longrightarrow}} C^{j}$ looks like

$$
B^{j}=\coprod_{\substack{i_{1}<i_{2}<\cdots<i_{j} \\ \text { some } i_{s}=r+1}} \overline{K[\Gamma]} /\left(\bar{N}_{i_{1}}+\bar{N}_{i_{2}}+\cdots+\bar{N}_{i_{j}}\right) .
$$


Thus by (12), after some straightforward fiddling with signs as in the proof $\left[\mathrm{Sta}_{8}\right.$, p. 72] of Reisner's theorem, $\mathscr{B}$ may be identified with the complex

$$
\mathscr{K}\left(\Gamma_{[d]-\{r+1\}}\right) /\left(\theta_{1}, \ldots, \theta_{r}\right)=\overline{\mathscr{K}}\left(\Gamma_{[d]-\{r+1\}}\right),
$$

shifted in degree by one (so $B^{0}=0, B^{1}=K\left[\Gamma_{[d]-\{r+1\}}\right] /\left(\theta_{1}, \ldots, \theta_{r}\right)$, etc.). Hence applying the induction hypothesis to $\Gamma_{[d]-\{r+1\}}$, it follows that $\mathscr{B}$ is exact except at $B^{1}$.

Now let $\mathscr{E}=\operatorname{ker}(\mathscr{C} \rightarrow \mathscr{D})=\operatorname{coker}(\mathscr{B} \rightarrow \mathscr{C})=\mathscr{C} / \mathscr{B}$. Thus we have two short exact sequences $0 \rightarrow \mathscr{E} \rightarrow \mathscr{C} \rightarrow \mathscr{D} \rightarrow 0$ and $0 \rightarrow \mathscr{B} \rightarrow \mathscr{C} \rightarrow \mathscr{E} \rightarrow 0$. The long exact sequence for cohomology (e.g., [H-S, p. 121]) shows that $H^{i}(\mathscr{D}) \cong$ $H^{i+1}(\mathscr{E}) \cong H^{i+2}(\mathscr{B}), i \geq 1$. Since $H^{j}(\mathscr{B})=0$ for $i \geq 2$, we get $H^{i}(\mathscr{D})=0$ for $i \geq 1$ as desired.

Proof of Theorem 4.6. Let $M=K[\Gamma]$. Consider the complex $\mathscr{K} / \theta \mathscr{K}$ of Lemma 4.9, i.e.,

$$
M / \theta M \stackrel{\delta^{0}}{\longrightarrow} \coprod_{i} M /\left(\theta M+N_{i}\right) \stackrel{\delta^{1}}{\longrightarrow} \cdots
$$

Let $L^{\prime}=\operatorname{ker}\left(\delta^{0}\right)$. Thus $L^{\prime}$ is the just the image of $N_{1} \cap \cdots \cap N_{d}$ in $M / \theta M$. But $N_{1} \cap \cdots \cap N_{d}=\left(\right.$ int $\Gamma$ ), so $L^{\prime}=L_{V}(\Gamma)$. Since $\mathscr{K} / \theta \mathscr{K}$ is exact by Lemma 4.9 , it follows that the Hilbert series $F\left(L_{V}(\Gamma), x\right)$ is given by

$$
\begin{aligned}
F\left(L_{V}(\Gamma), x\right)= & F(M / \theta M), x)-F\left(\coprod_{i} M /\left(N_{i}+\theta M\right), x\right) \\
& +\cdots+(-1)^{d} F\left(\coprod_{i} M /\left(N_{1}+\cdots+N_{d}+\theta M\right), x\right) \\
= & \sum_{W \subseteq V}(-1)^{\#(V-W)} F\left(K\left[\Gamma_{W}\right] / \theta, x\right) .
\end{aligned}
$$

But since $K\left[\Gamma_{W}\right]$ is Cohen-Macaulay and $\theta$ is an h.s.o.p. of degree one of $K\left[\Gamma_{W}\right]$ (except for irrelevant elements $\theta_{i}$ which annihilate $K\left[\Gamma_{W}\right]$ ), we have

$$
F\left(K\left[\Gamma_{W}\right] / \theta, x\right)=h\left(\Gamma_{W}, x\right) .
$$

By (4) it follows that $F\left(L_{V}(\Gamma), x\right)=\ell_{V}(\Gamma, x)$, completing the proof.

Note. Let $I$ denote the kernel of the map

$$
K[\Gamma] \longrightarrow \coprod_{i} K[\Gamma] / N_{i} \text {. }
$$

In view of (14) it is natural to ask what is the Hilbert series of $I / \theta I$. But clearly $I=($ int $\Gamma)$, so by $\left[\mathrm{Sta}_{8}\right.$, Chapter II.7] we have

$$
F(I / \theta I, x)=h_{d}+h_{d-1} x+\cdots+h_{0} x^{d},
$$


where $h(\Gamma)=\left(h_{0}, \ldots, h_{d}\right)$. Thus the exact sequence

$$
0 \rightarrow(\text { int } \Gamma) \rightarrow K[\Gamma] \rightarrow \coprod_{i} K[\Gamma] / N_{i}
$$

does not stay exact when we mod out by $\theta$, since

$$
(\text { int } \Gamma) /\left(\theta_{1}(\text { int } \Gamma)+\cdots+\theta_{d}(\text { int } \Gamma)\right) \neq L_{V}(\Gamma) \text {. }
$$

Note. For the class of shellable (or more generally, partitionable) simplicial complexes, there is a simple combinatorial interpretation of their $h$-vectors which shows that they are nonnegative (see [ $\mathrm{Sta}_{5}$, Proposition 3.6] for a more general result). We have been unable to find a similar result for local $h$-vectors, say for shellable quasi-geometric subdivisions of a simplex.

Note. A result related to Corollary 4.7 appears in [G-Z-K]. Comparing their definition of the "combinatorial Newton number" $N C_{V}(\Gamma)$ of a triangulation $\Gamma$ of the simplex $2^{V}$ with our equation (4) shows that $N C_{V}(\Gamma)=\ell_{V}(\Gamma, 1)$. They show that $N C_{V}(\Gamma) \geq 0$ when $\Gamma$ is geometric, essentially the same way in which we prove Theorem 5.2 below. In [G-Z-K] there is also a discussion of "Newton number" and "relative Newton number." This topic is closely related to the paper [B-M] and to our Example 7.13.

Theorem 3.2 and Corollary 4.7 lead immediately to our original goal of showing that under certain circumstances the $h$-vector of a Cohen-Macaulay complex increases under subdivision.

4.10. Theorem. Suppose $\Delta$ is a simplicial complex and $\Delta^{\prime}$ a quasi-geometric simplicial subdivision of $\Delta$. Then $h\left(\Delta^{\prime}\right) \geq h(\Delta)$ provided one of the following successively weaker conditions holds:

(a) $\Delta$ is Cohen-Macaulay.

(b) $\Delta$ is Buchsbaum. This means that $\Delta$ is pure and the link of every vertex $v$ (or equivalently, of every nonempty face) is Cohen-Macaulay. (Characterizations of Buchsbaum complexes appearing in [Sc; S-V, Theorem 2.4, p. 116; Sta , Chapter II, Theorem 8.1] omitted the condition that $\Delta$ is pure. The necessity for this condition was pointed out in [Mi, Remark, p. 251].)

(c) $\Delta$ is pure, and for every $F \in \Delta$ with $\# F \geq 2$ we have $h\left(l k_{\Delta} F, x\right) \geq 0$ (i.e., $h_{i}\left(l k_{\Delta} F\right) \geq 0$ for all $i$ ).

(d) $\Delta$ is pure, and for every $\varnothing \neq F \in \Delta$ with $\ell_{F}\left(\Delta_{F}^{\prime}, x\right) \neq 0$ we have $h\left(l k_{\Delta} F, x\right) \geq 0$.

Proof. We have $(\mathrm{c}) \Rightarrow(\mathrm{d})$ since $\ell_{F}\left(\Delta_{F}^{\prime}, x\right)=0$ when $\# F=1$ (so that $\Delta_{F}^{\prime}$ is just a single vertex). We have (b) $\Rightarrow$ (c) since if $\mathrm{lk}_{\Delta} F$ is Cohen-Macaulay, then $h\left(\mathrm{lk}_{\Delta} F, x\right) \geq 0$. We have (a) $\Rightarrow$ (b) immediately from the definition of Cohen-Macaulay and Buchsbaum complexes.

Hence assume $(\mathrm{d})$. Since $\ell_{\phi}(\phi, x)=1$ and $\mathrm{lk}_{\Delta} \varnothing=\Delta$, the term indexed by $F=\varnothing$ in (9) is just $h(\Delta, x)$. By Corollary 4.7 and (d), all the terms $F \neq \varnothing$ in the right-hand side of (9) are nonnegative. Hence $h\left(\Delta^{\prime}, x\right) \geq h(\Delta, x)$ as desired. 
4.11. Conjecture. The hypothesis that $\Delta^{\prime}$ is quasi-geometric is not needed in Theorem 4.10 (at least in case (a)).

Note. There is an alternative condition for which one can show that $h\left(\Delta^{\prime}\right) \geq$ $h(\Delta)$. Define a pure simplicial complex $\Delta$ to be partitionable if it can be written as a disjoint union of intervals $[F, G]:=\{J \in \Delta: F \subseteq J \subseteq G\}$, where $G$ is a facet of $\Delta$. For instance, shellable simplicial complexes are partitionable, and it is conjectured that all Cohen-Macaulay complexes are partitionable. Using the techniques of [ $\mathrm{Sta}_{10}, \S 5$ ], Kalai and this author showed (unpublished) that if $\Delta^{\prime}$ is a quasi-geometric simplicial subdivision of a partitionable simplicial complex $\Delta$, then $h\left(\Delta^{\prime}\right) \geq h(\Delta)$.

4.12. Example. We show that $h\left(\Delta^{\prime}\right) \geq h(\Delta)$ does not hold for every pure $\Delta$ and subdivision $\Delta^{\prime}$. By Corollary $4.10(\mathrm{c})$ and the fact that $h(\Gamma, x) \geq 0$ when $\operatorname{dim} \Gamma \leq 0$, the smallest possible value of $\operatorname{dim} \Delta$ is 3 . Let $\Delta$ have maximal faces 1234 and 1256. Add a new vertex 7 on the edge 12 , and let $\Delta^{\prime}$ be the subdivision of $\Delta$ with maximal faces $1347,2347,1567,2567$. Then $h(\Delta, x)=1+2 x-x^{2}$ and $h\left(\Delta^{\prime}, x\right)=1+3 x+x^{2}-x^{3}$, so $h_{3}\left(\Delta^{\prime}\right)<h_{3}(\Delta)$.

Condition (d) in Theorem 4.10 suggests the following problem.

4.13. Problem. Find a "nice" characterization or description of all quasigeometric simplicial subdivisions $\Gamma$ of the simplex $2^{V}$ such that $\ell_{V}(\Gamma, x)=$ 0 .

The following result gives a solution of sorts to Problem 4.13, but it would be preferable to have a more explicit characterization.

4.14. Proposition. Let $\Gamma$ be a quasi-geometric simplicial subdivision of $2^{V}$, with $\# V=d$. Then $\ell_{V}(\Gamma, x)=0$ if and only if the number of even-dimensional faces of $\Gamma$ of excess 0 is equal to the number of odd-dimensional faces of excess 0 .

Proof. By Corollary 4.7 we have $\ell_{V}(\Gamma, x)=0$ if and only if $\ell_{V}(\Gamma, 1)=0$. The result now follows by putting $x=1$ in equation (5).

Thus, for instance, if \#V=2 or 3 , then $\ell_{V}(\Gamma, x)=0$ if and only if $\Gamma$ has no interior vertices. If $\# V=4$ or 5 , then $\ell_{V}(\Gamma, x)=0$ if and only if $\Gamma$ has no interior vertices and the number of interior edges of $\Gamma$ is equal to the number of vertices of $\Gamma$ whose support is a face with $d-1$ vertices. (See Example $2.3(\mathrm{c}, \mathrm{d}, \mathrm{g})$.) Two other instances where $\ell_{V}(\Gamma, x)=0$ are the following.

(a) Let $v \in V$. Let $\Gamma^{\prime}$ be any simplicial subdivision of $2^{V-\{v\}}$. Let $\Gamma$ be the simplicial join $v * \Gamma^{\prime}$ (i.e., the cone over $\Gamma^{\prime}$ with apex $v$ ). Then it is easy to see that $\ell_{V}(\Gamma, x)=0$.

(b) Suppose $d$ is odd and $e(v)=0$ or 1 for every vertex $v$ of $\Gamma$. Then it follows from the proof of Proposition 3.4 that $\ell_{V}(\Gamma, x)=0$. More generally, if $2 e(G)<d$ for all $G \in \Gamma$, then $\ell_{V}(\Gamma, x)=0$. 
Note. Putting $x=1$ in equation (5) and using Corollary 4.7 shows that for any quasi-geometric simplicial subdivision $\Gamma$ of $2^{V}$, we have

$$
\sum_{\substack{G \in \Gamma \\ e(G)=0}}(-1)^{d-\# G} \geq 0 .
$$

Is there a proof of this inequality avoiding Corollary 4.7 ?

An interesting property of quasi-geometric subdivisions with local $h$-vector equal to 0 is given by the next proposition.

4.15. Proposition. Let $\Gamma$ be a quasi-geometric simplicial subdivision of $2^{V}$ with $\ell_{V}(\Gamma, x)=0$. If $\Gamma^{\prime}$ is any quasi-geometric simplicial subdivision of $2^{V}$ with $\partial \Gamma=\partial \Gamma^{\prime}$, then $h\left(\Gamma^{\prime}\right) \geq h(\Gamma)$. In other words, $\Gamma$ minimizes $h\left(\Gamma^{\prime}\right)$ among all quasi-geometric simplicial subdivisions $\Gamma^{\prime}$ of $2^{V}$ with the same boundary as $\Gamma$. Proof. By (3) we have

$$
h\left(\Gamma^{\prime}, x\right)-h(\Gamma, x)=\ell_{V}\left(\Gamma^{\prime}, x\right),
$$

which is nonnegative by Corollary 4.7 .

Proposition 4.15 suggests the following problem, which is of course closely related to Problem 4.13.

4.16. Definition. (a) Given a quasi-geometric simplicial subdivision $\Gamma^{\prime}$ of the boundary $\partial 2^{V}$ of the simplex $2^{V}$, when does there exist a quasi-geometric simplicial subdivision $\Gamma$ of $2^{V}$ satisfying $\partial \Gamma=\Gamma^{\prime}$ and $\ell_{V}(\Gamma, x)=0$ ?

(b) More generally, given $\Gamma^{\prime}$ find the minimum values of $h_{i}(\Gamma)$ or $f_{i}(\Gamma)$ among all quasi-geometric simplicial subdivisions $\Gamma$ of $2^{V}$ satisfying $\partial \Gamma=$ $\Gamma^{\prime}$.

Part (b) above is probably hopeless, but (a) may be more tractable. The next example shows that (a) does not have the trivial answer "always."

4.17. Example. It is easy to see that if $\# V \leq 3$ then the answer to Problem 4.16(a) is "always." We give an example where $\# V=4$ and $\Gamma$ does not exist. Let $V=\left\{x_{1}, x_{2}, x_{3}, x_{4}\right\}$. Add a new vertex $x_{i j}$ inside each edge $x_{i} x_{j}(1 \leq i<j \leq 4)$. Subdivide each facet $x_{i} x_{j} x_{k}$ of $\partial 2^{V}$ so the maximal faces are $x_{i} x_{i j} x_{i k}, x_{j} x_{i j} x_{j k}, x_{k} x_{i k} x_{j k}$, and $x_{i j} x_{i k} x_{j k}$. We claim that this subdivision $\Gamma^{\prime}$ of $\partial 2^{V}$ cannot be extended to a subdivision $\Gamma$ of $2^{V}$ such that $\partial \Gamma=\Gamma^{\prime}$ and $\ell_{V}(\Gamma, x)=0$. If on the contrary $\partial \Gamma=\Gamma^{\prime}$ and $\ell_{V}(\Gamma, x)=0$, then one computes from (3) that $h(\Gamma, x)=1+6 x$. Thus $\Gamma$ has seven facets. Since the 2-faces of $\Gamma$ have no interior vertices, it follows from the paragraph after Proposition 4.14 that $\Gamma$ has no interior vertices or edges. Since three edges of $\Gamma$ meet at each vertex $x_{i}$ of $2^{V}$, exactly one facet $F_{i}$ of $\Gamma$ contains $x_{i}$. When we remove the open star of each $x_{i}$ from $\Gamma$, we will remove the four facets $F_{i}$ and no other facets, and will remain (since each edge of $2^{V}$ has one interior vertex in $\Gamma$ ) with an octahedron triangulated with three facets. But a 
little thought shows that there is no way to arrange (even abstractly) for three tetrahedra to triangulate an octahedron, so $\Gamma$ does not exist.

Theorems 3.3 and 4.6 show that the graded module $L_{V}(\Gamma)=L_{0} \oplus \cdots \oplus L_{d}$ satisfies $\operatorname{dim}_{K} L_{i}=\operatorname{dim}_{K} L_{d-i}$, for $0 \leq i \leq d$. This suggests that $L_{V}(\Gamma)$ is a "Gorenstein module." Since $L_{V}(\Gamma)$ has finite length, this means that the (Matlis) dual

$$
L_{V}(\Gamma)^{*}=\operatorname{Hom}_{K}\left(L_{V}(\Gamma), K\right)
$$

is isomorphic (up to a shift in grading) to $L_{V}(\Gamma)$ (as an $R_{\Gamma}$-module or $K[\Gamma]$ module). Indeed this is the case, as follows from the following result due to D. Eisenbud (private communication). Since we will not use this result later and it gives nothing new about the local $h$-vector $\ell_{V}(\Gamma)$ itself, we omit the proof.

4.18. Proposition. Let $A$ be a Cohen-Macaulay ring possessing a canonical module $\Omega_{A}$ which is an ideal of $A$. Let $\theta_{1}, \ldots, \theta_{d}$ be a maximal A-sequence. Then the image of $\Omega_{A}$ in $A /\left(\theta_{1}, \ldots, \theta_{d}\right)$ is a Gorenstein A-module.

4.19. Corollary. $L_{V}(\Gamma)$ is a Gorenstein $K[\Gamma]$-module (or $R_{\Gamma}$-module).

Proof. Follows from Proposition 4.18 and the fact $\left[\mathrm{Sta}_{8}\right.$, Theorem 7.3] that (int $\Gamma$ ) is the canonical module of $K[\Gamma]$.

Note. Let $M$ be any module of finite length (over a commutative noetherian ring $A$ ). Let $M^{*}$ be the canonical module (or Matlis dual) of $M$. Let $\varphi$ : $M^{*} \rightarrow M$ be a homomorphism. It is natural to ask whether Proposition 4.18 can be generalized to the statement that $\varphi\left(M^{*}\right)$ is Gorenstein. A counterexample, however, has been provided by $\mathrm{C}$. Huneke.

Theorem 4.6 leads in certain circumstances to an interesting refinement of the local $h$-vector. Suppose $G$ is a group of automorphisms of the simplex $2^{V}$ (so $G$ is isomorphic to a subgroup of the symmetric group $\mathscr{S}_{d}$, where $\# V=d)$, and that $\Gamma$ is a $G$-compatible quasi-geometric subdivision of $2^{V}$ (i.e., each $w \in G$ induces an automorphism of $\Gamma$ in the obvious way). Suppose $\theta_{1}, \ldots, \theta_{d} \in K[\Gamma]_{1}$ is a special h.s.o.p. for $K[\Gamma]$ such that the vector space $\operatorname{span}_{K}\left\{\theta_{1}, \ldots, \theta_{d}\right\}$ is $G$-invariant. It is easy to see that $G$ then acts in a natural way on $L_{V}(\Gamma)$, and that this action is degree-preserving. Assume char $K$ is relatively prime to $\# G$, so the $G$-module $L_{i}=L_{V}(\Gamma)_{i}$ is a direct sum of irreducible modules. Let $\hat{G}$ be the set of irreducible characters of $G$, and for $\chi \in \hat{G}$ let $m_{\chi}(i)$ be the multiplicity of $\chi$ in the action of $G$ on $L_{i}$. Then

$$
\ell_{i}=\sum_{\chi \in \hat{G}} m_{\chi}(i)(\operatorname{deg} \chi),
$$

so we have obtained a "refinement" of the numbers $\ell_{i}$. (For a similar situation, see $\left.\left[\mathrm{Sta}_{7}, \S 1\right].\right)$

We give one example illustrating a group action on $L_{i}$; viz., let $\Gamma=\operatorname{sd}\left(2^{V}\right)$ as in Example 2.3(i). Let $G$ be the full automorphism group of $2^{V}$, so $G \cong \mathscr{S}_{d}$, 
the symmetric group of degree $d$. We assume familiarity with the irreducible characters of $\mathscr{S}_{d}$, and their connection with symmetric functions as explained in [ $\mathrm{Ma}_{2}$, Chapter 1.7]. Let $\varepsilon_{1}, \ldots, \varepsilon_{d}$ be the unit coordinate vectors in $\mathbf{R}^{d}$. Assume that the simplex $2^{V}$ is geometrically realized in $\mathbf{R}^{d}$ as the Euclidean simplex $\sigma=\operatorname{conv}\left\{\varepsilon_{1}, \ldots, \varepsilon_{d}\right\}$, and that $\operatorname{sd} \sigma$ is the usual Euclidean barycentric subdivision. Let $\varepsilon_{1}^{*}, \ldots, \varepsilon_{d}^{*}: \mathbf{R}^{d} \rightarrow \mathbf{R}$ be the dual basis to $\left\{\varepsilon_{1}, \ldots, \varepsilon_{d}\right\}$, so $\varepsilon_{i}^{*}\left(\varepsilon_{j}\right)=\delta_{i j}$. If sd $\sigma$ has vertex set $V^{\prime}$, then define $\theta_{i} \in \mathbf{R}[\Gamma]_{1}$ by

$$
\theta_{i}=\sum_{v \in V^{\prime}} \varepsilon_{i}^{*}(v) v
$$

Clearly $\theta_{1}, \ldots, \theta_{d}$ is a special h.s.o.p., and the $\operatorname{space} \operatorname{span}_{\mathbf{R}}\left\{\theta_{1}, \ldots, \theta_{d}\right\}$ is $G$ invariant. Let $E_{i d}=E_{i d}(t)=E_{i d}\left(t_{1}, t_{2}, \ldots\right)$ be the characteristic (in the sense of $\left.\left[\mathrm{Ma}_{2}, \mathrm{p} .61\right]\right)$ of the action of $\mathscr{S}_{d}$ on $R_{i}$, where $R=\mathbf{R}[\Gamma] /\left(\theta_{1}, \ldots, \theta_{d}\right)$ $=R_{0} \oplus R_{1} \oplus \cdots \oplus R_{d}$. Thus $E_{i d}(t)$ is a symmetric function in the variables $t=\left(t_{1}, t_{2}, \ldots\right)$ which is homogeneous of degree $d$. It follows from [ $\operatorname{Sta}_{12}$, Proposition 12] and its proof that

$$
\sum_{d \geq 0} \sum_{i \geq 0} E_{i d} x^{i}=\frac{\sum_{j \geq 0} s_{j}}{1-x \sum_{j \geq 2}\left(1+x+\cdots+x^{j-2}\right) s_{j}},
$$

where $s_{j}=s_{j}(t)$ is the Schur function indexed by the partition $(j)$ (so also $s_{j}=h_{j}$ in the notation of $\left.\left[\mathrm{Ma}_{2}\right]\right)$. (We do not need another variable in (16) keeping track of the index $d$ since $E_{i d}$ is homogeneous of degree $d$.)

4.20. Proposition. Let $E_{i d}^{\prime}=E_{i d}^{\prime}(t)$ denote the characteristic of the action of $\mathscr{S}_{d}$ on $L_{i}=L_{V}(\Gamma)_{i}$, where $\Gamma=s d V$ as above, and where $L_{V}(\Gamma)$ is defined with respect to the special h.s.o.p. $\theta_{1}, \ldots, \theta_{d}$ given by (15). Then

$$
\sum_{d \geq 0} \sum_{i \geq 0} E_{i d}^{\prime} x^{i}=\frac{1}{1-x \sum_{j \geq 2}\left(1+x+\cdots+x^{j-2}\right) s_{j}} .
$$

Proof (sketch). Let $V=\left\{x_{1}, \ldots, x_{d}\right\}$. In the complex (11) choose $K=\mathbf{R}$, $M=\mathbf{R}[\Gamma], N_{i}$ to be as in the proof of Theorem 4.6, and $\theta_{1}, \ldots, \theta_{d}$ to be given by (15). For $0 \leq r \leq d$ define an action of $\mathscr{S}_{d}$ on the module

$$
M_{r d}:=\coprod_{1 \leq i_{1}<\cdots<i_{r} \leq d} \mathbf{R}[\Gamma] /\left(N_{i_{1}}+\cdots+N_{i_{r}}\right) \cong \coprod_{\substack{s \subseteq V=\left\{x_{1}, \ldots, x_{d}\right\} \\ \# S=r}} \mathbf{R}\left[\Gamma_{V-S}\right]
$$

as follows. Let $u=y_{1}^{a_{1}} \cdots y_{k}^{a_{k}}$ be a monomial in $\mathbf{R}\left[\Gamma_{V-S}\right]$. Thus the support of $u$ is a face of $\Gamma_{V-S}$. If $w \in \mathscr{S}_{d}$, then set

$$
w \cdot u=(\operatorname{sgn} w)\left(w \cdot y_{1}\right)^{a_{1}} \cdots\left(w \cdot y_{k}\right)^{a_{k}} \in \Gamma_{w \cdot(V-S)} .
$$

One checks that (19) does indeed define an action of $\mathscr{S}_{d}$ on (11), and that this action commutes with the coboundary operator in (11). The vector space $\operatorname{span}_{\mathbf{R}}\left\{\theta_{1}, \ldots, \theta_{d}\right\} \subset K[\Gamma]$ is $\mathscr{S}_{d}$-invariant, so we obtain an induced action of $\mathscr{S}_{d}$ on the complex (11). 
As explained in the proof of Theorem 4.6, the complex (11) is acyclic. For a graded vector space $W=W_{0} \oplus W_{1} \oplus \cdots$ with each $W_{i}$ a finite-dimensional $\mathscr{S}_{d}$-module, write $\operatorname{ch}(W, x)$ for the power series

$$
\operatorname{ch}(W, x)=\sum_{n \geq 0}\left(\operatorname{ch} W_{i}\right) x^{i},
$$

where ch $W_{i}$ denotes the characteristic of the action of $\mathscr{S}_{d}$ on $W_{i}$. It follows from the proof of Theorem 4.6 (including the exactness of (11)) that $\mathscr{S}_{d}$ acts on $L_{V}(\Gamma)$ so that

$$
\operatorname{ch}\left(L_{V}(\Gamma), x\right)=\sum_{i \geq 0} E_{i d}^{\prime} x^{i}=\sum_{r \geq 0}(-1)^{r} \operatorname{ch}\left(M_{r d} / \theta M_{r d}, x\right),
$$

where $M_{r d}$ is given by (18).

Now a not-so-difficult combinatorial argument (really the crux of the proof) shows that

$$
\operatorname{ch}\left(M_{r d} / \theta M_{r d}, x\right)=e_{r} \cdot \operatorname{ch}\left(M_{0, d-r} / \theta M_{0, d-r}, x\right),
$$

where $e_{r}$ denotes the $r$ th elementary symmetric function. Note that $M_{0, d-r}$ is just $\mathbf{R}\left[\Gamma^{(d-r)}\right]$, where the superscript $(d-r)$ indicates that $\Gamma^{(d-r)}=\operatorname{sd} 2^{V}$ for $\# V=d-r$. Thus

$$
\operatorname{ch}\left(M_{0, d-r} / \theta M_{0, d-r}, x\right)=\sum_{i \geq 0} E_{i d} x^{i}
$$

so

$$
\begin{aligned}
\sum_{d \geq 0} \sum_{i \geq 0} E_{i d}^{\prime} x^{i} & =\sum_{d \geq 0} \sum_{r \geq 0}(-1)^{r} e_{r} \sum_{i \geq 0} E_{i d} x^{i} \\
& =\left(\sum_{r \geq 0}(-1)^{r} e_{r}\right) \sum_{d \geq 0} \sum_{i \geq 0} E_{i d} x^{i} .
\end{aligned}
$$

The proof follows from (16) and the well-known (and easy) formula

$$
\sum_{r \geq 0}(-1)^{r} e_{r}=\left(\sum_{j \geq 0} s_{j}\right)^{-1}
$$

The generating function (17) first appeared in $\left[\mathrm{Sta}_{12}\right.$, Proposition 13] in a purely combinatorial setting. Letting $R_{\lambda}(x)$ denote the coefficient of $s_{\lambda}$ when (17) is expanded in terms of Schur functions, it was conjectured in [ $\mathrm{Sta}_{12}$ ] that $R_{\lambda}(x)$ is a unimodal polynomial. This conjecture was proved by Brenti $\left[\mathrm{Br}_{1}\right.$ ] and later Stembridge [Ste]. It can also be proved by using a suitable refinement of Theorem 5.2. The generating function (17) also appears in [G-K$\mathrm{Z}]$ in a completely different context. Its square may be regarded as the generating function for the degree of the hyperdeterminant. We do not know whether the appearance of (17) in our work and in [G-K-Z] is just a coincidence. 
We conclude this section by mentioning a generalization of the local $h$-vector $\ell_{V}(\Gamma)$. If $\Delta$ is any $(d-1)$-dimensional simplicial complex and $\Delta^{\prime}$ a simplicial subdivision of $\Delta$, then we can define in analogy with equation (4) a "local $h$-polynomial" $\ell_{\Delta}\left(\Delta^{\prime}, x\right)$ by

$$
\ell_{\Delta}\left(\Delta^{\prime}, x\right)=\sum_{F \in \Delta}(1)^{d-|F|} h\left(\Delta_{F}^{\prime}, x\right) .
$$

We do not, however, know of any sort of "local" interpretation of $\ell_{\Delta}\left(\Delta^{\prime}, x\right)$. It is easy to find examples (even when $\Delta$ triangulates a ball) where $\ell_{\Delta}\left(\Delta^{\prime}, x\right) \neq$ $x^{d} \ell_{\Delta}\left(\Delta^{\prime}, 1 / x\right)$. We do, however, have the following result.

4.21. Proposition. Let $\Delta^{\prime}$ be a quasi-geometric subdivision of the CohenMacaulay simplicial complex $\Delta$. Then $\ell_{\Delta}\left(\Delta^{\prime}, x\right) \geq 0$.

Proof. We have

$$
\begin{aligned}
\ell_{\Delta}\left(\Delta^{\prime}, x\right) & =\sum_{F \in \Delta}(-1)^{d-|F|} h\left(\Delta_{F}^{\prime}, x\right) \\
& =\sum_{F \in \Delta}(-1)^{d-|F|} \sum_{G \subseteq F} \ell_{G}\left(\Delta_{G}^{\prime}, x\right) \\
& =\sum_{G \in \Delta} \ell_{G}\left(\Delta_{G}^{\prime}, x\right) \sum_{F \supseteq G}(-1)^{d-|F|} \\
& =\sum_{G \in \Delta} \ell_{G}\left(\Delta_{G}^{\prime}, x\right)(-1)^{d-|G|} \tilde{\chi}\left(\mathrm{k}_{\Delta} G\right) .
\end{aligned}
$$

When $\Delta$ is Cohen-Macaulay we have $(-1)^{d-|G|} \tilde{\chi}\left(\mathrm{lk}_{\Delta}, G\right) \geq 0$. Moreover, if $\Delta^{\prime}$ is a quasi-geometric subdivision of $\Delta$, then $\Delta_{G}^{\prime}$ is a quasi-geometric subdivision of $2^{G}$ for any $G \in \Delta$. Thus the proof follows from Corollary 4.7.

Of course the hypothesis that $\Delta$ is Cohen-Macaulay in Proposition 4.21 can be relaxed somewhat. For instance, we need only assume that $(-1)^{d-|G|} \tilde{\chi}\left(\mathrm{kk}_{\Delta} G\right)$ $\geq 0$ for every face $G \in \Delta$ such that $\operatorname{dim} G \neq 0$.

\section{UNIMODALITY OF REGULAR LOCAL $h$-VECTORS}

For our fourth basic property of local $h$-vectors, we need the concept of "regular subdivision" (or "regular triangulation") as discussed, for instance, in [B-F-S, L].

5.1. Definition. Let $L=L(\mathscr{P})$ be the face lattice of a convex $(d-1)$-polytope $\mathscr{P}$. An abstract polyhedral subdivision $\Gamma$ of $L$ (or of $\mathscr{P}$ ) is called regular if $\Gamma$ can be geometrically realized in $\mathbf{R}^{d-1}$ as a Euclidean subdivision of a convex polytope $\mathscr{P}$ whose face lattice is $L$ (so for every $F \in \Gamma$, the geometric realization $|F| \subseteq \mathscr{P}$ is a convex polytope) with the following property: There exists a function $\omega: \mathscr{P} \rightarrow \mathbf{R}$ which is (a) piecewise-linear, i.e., $\omega$ is linear on each face $|F|$, and (b) strictly convex, i.e., $\omega$ is convex $(\omega(\lambda u+(1-\lambda) v) \geq$ $\lambda \omega(u)+(1-\lambda) \omega(v)$ for all $u, v \in|\Gamma|$ and $0 \leq \lambda \leq 1)$ and is a different linear 
function on each facet $((d-1)$-face) of $|\Gamma|$. (One can think that there is a strictly convex polyhedral surface in $\mathbf{R}^{d}$ which projects to the subdivision of $|\Gamma|$ in $\mathbf{R}^{d-1}$.)

Note. The definition of regular subdivision in [B-F-S] or [L] applies only to the geometric, and not the abstract, situation. We have actually extended the definition to abstract complexes by defining $\Gamma$ to be regular if it possesses a regular (in the sense of [B-F-S] or [L]) geometric realization. Also in [B-F-S] and $[\mathrm{L}]$ the vertices of the subdivision are given in advance, but this is not relevant to us here.

Note that a regular subdivision $\Gamma$ of $\mathscr{P}$ (as in Definition 5.1) is geometric and hence quasi-geometric.

5.2. Theorem. Let $\Gamma$ be a regular simplicial subdivision of the simplex $2^{V}$, where $|V|=d$. Then the local h-vector $\ell_{V}(\Gamma)=\left(\ell_{0}, \ldots, \ell_{d}\right)$ is unimodal; i.e. (since $\left.\ell_{i}=\ell_{d-i}\right)$,

$$
\ell_{0} \leq \ell_{1} \leq \cdots \leq \ell_{\lfloor d / 2\rfloor}
$$

Proof. Let $H$ be an affine hyperplane (which we identify with $\mathbf{R}^{d-1}$ ) in $\mathbf{R}^{d}$ which does not contain the origin. Let $\Gamma^{\prime}$ be a regular geometric realization in $H$ of $\Gamma$, as defined in Definition 5.1. Thus $\Gamma^{\prime}$ is a collection of Euclidean simplices $\sigma$ (with suitable properties), and $\left|\Gamma^{\prime}\right|=\bigcup_{\sigma \in \Gamma^{\prime}} \sigma$ is a Euclidean simplex. Small perturbations of the vertices $v$ of $\Gamma^{\prime}$ which preserve the faces $F$ of $\left|\Gamma^{\prime}\right|$ on which the vertices $v$ of $\Gamma^{\prime}$ lie will not affect the combinatorial structure or the regularity (since all faces are simplices) of $\Gamma^{\prime}$, so we can assume that the vertices have rational coordinates. (First perturb to rational vertices those vertices of $\Gamma^{\prime}$ which are vertices of $\left|\Gamma^{\prime}\right|$.)

For each face $F$ of the simplex $\left|\Gamma^{\prime}\right|$, let $C(F)$ denote the cone of all rays through $F$ with endpoint at the origin. The set $\Sigma_{1}$ of all such cones forms a rational fan. Similarly let $\Sigma_{2}$ be the rational fan of all cones $C(\sigma)$, where $\sigma \in \Gamma^{\prime}$. Let $X_{1}$ and $X_{2}$ be the (complex) toric varieties corresponding to the fans $\Sigma_{1}$ and $\Sigma_{2}$, as defined e.g. in [Dan].

Since $\Sigma_{2}$ is a subdivision of $\Sigma_{1}$, we have a corresponding proper algebraic map $f: X_{2} \rightarrow X_{1}$ [Dan, 5.5.1]. Thus we can apply the decomposition theorem for intersection homology, as described in [Mac, Theorem 1.12]. We take the stratification $X_{1}=\bigcup_{i}\left(X_{1}\right)_{i}$ of $X_{1}$ where $\left(X_{1}\right)_{i}$ is the inverse image of the interior of the $i$-dimensional faces of $\left|\Gamma^{\prime}\right|$ under the moment map. The local systems $L_{i}$ can be all taken to be trivial, and there is a standard choice of the smoothly enclosed subsets $A \subseteq B \subseteq X_{1}$ in $X_{1}$. (Actually, $A$ can be done away with in the present situation; there is no need to consider relative intersection homology.) The intersection homology $\mathrm{IH}\left(\mathrm{X}_{2}\right)$ of $\mathrm{X}_{2}$ with compact supports coincides with the singular homology $H\left(X_{2}\right)$ with compact supports (over $\mathbf{C}$, say) since $\Gamma$ is simplicial, and is given by

$$
\begin{aligned}
\operatorname{dim} I H_{2 i}\left(X_{2}\right) & =h_{i}(\Gamma), \\
I H_{2 i+1}\left(X_{2}\right) & =0 .
\end{aligned}
$$


The decomposition theorem shows that the polynomials $\phi^{i}$ in the statement of this theorem satisfy

$$
h(\Gamma, x)=\sum_{\substack{F \subseteq V \\ \operatorname{dim} F=i}} \phi^{i}(x) .
$$

Since this formula is true for $V$ replaced with any $F \subseteq V$ and $\Gamma$ replaced with $\Gamma_{F}$, comparing with (3) yields

$$
\phi^{i}(x)=\sum_{\substack{F \subseteq V \\ \operatorname{dim} F=i}} \ell_{F}\left(\Gamma_{F}, x\right) .
$$

In particular, $\phi^{d-1}(x)=\ell_{V}(\Gamma, x)$.

The hypothesis that $\Gamma^{\prime}$ is a regular subdivision of $\left|\Gamma^{\prime}\right|$ implies that $f$ is projective. (In fact, $f$ is projective if and only if $\Gamma^{\prime}$ is regular.) By the hard Lefschetz property of the decomposition theorem, we have that $\phi^{d-1}(x)=$ $\ell_{V}(\Gamma, x)$ is unimodal, as desired.

Note. We do not need projectivity of $f$ to conclude that $\phi^{d-1}(x)$ has nonnegative coefficients. Thus we have another (and much more difficult) proof of Corollary 4.7 when $\Gamma$ is a geometric simplicial subdivision of $2^{V}$.

5.3. Corollary. Let $\Delta$ be a pure simplicial complex such that for every face $F \in$ $\Delta$ the $h$-vector $\left(h_{0}, \ldots, h_{d-\# F}\right)$ of $l k_{\Delta} F$ is symmetric $\left(h_{i}=h_{d-\# F-i}\right.$ for all $i)$ and unimodal $\left(h_{0} \leq h_{1} \leq \cdots \leq h_{\left\lfloor\frac{1}{2}(d-\# F)\right\rfloor}\right.$, assuming symmetry). (By [ Sta 6 ] the boundary complex of a simplicial convex polytope satisfies this condition. For a generalization, see [Ka, §8] and $\left[\mathrm{Sta}_{13}\right.$, Corollary 2.4].) Let $\Delta^{\prime}$ be a simplicial subdivision of $\Delta$ such that for all $F \in \Delta$, the restriction $\Delta_{F}^{\prime}$ is a regular subdivision of $2^{F}$. Then

$$
h_{i}(\Delta)-h_{i-1}(\Delta) \leq h_{i}\left(\Delta^{\prime}\right)-h_{i-1}\left(\Delta^{\prime}\right), \quad 0 \leq i \leq\lfloor d / 2\rfloor .
$$

Proof. Consider equation (9). By hypothesis each polynomial $h\left(\mathrm{lk}_{\Delta} F, x\right)$ is symmetric and unimodal, with center of symmetry at $\frac{1}{2}(d-\# F)$. By Theorem $3.3, \ell_{F}\left(\Delta_{F}^{\prime}, x\right)$ is symmetric, with center of symmetry $\frac{1}{2}(\# F)$. By Theorem 5.2, $\ell_{F}\left(\Delta_{F}^{\prime}, x\right)$ is unimodal. Hence the product $\ell_{F}\left(\Delta_{F}^{\prime}, x\right) h\left(\mathrm{k}_{\Delta} F, x\right)$ is symmetric about $d / 2$, and by e.g. [ $\operatorname{Sta}_{12}$, Proposition 1] is unimodal. The term indexed by $F=\varnothing$ in (9) is just $h(\Delta, x)$, and the proof follows.

Note. As in Theorem 4.10, the conclusion (21) to Corollary 5.3 holds if we replace the symmetry and unimodality of $h\left(\mathrm{lk}_{\Delta} F\right)$ for all $F \in \Delta$ by symmetry and unimodality for all $F \neq \varnothing$ for which $\ell_{F}\left(\Delta_{F}^{\prime}, x\right) \neq 0$, and just symmetry for $h(\Delta, x)$ itself (the case $F=\varnothing$ ).

5.4. Conjecture. Theorem 5.2 continues to hold when $\Gamma$ is a quasi-geometric simplicial subdivision of $2^{V}$. (Example 2.3(h) shows that Theorem 5.2 fails for arbitrary simplicial subdivisions of $2^{V}$.) 


\section{PART II. GENERALIZATIONS}

\section{KERNELS AND $\kappa$-ACCEPTABILITY}

It is natural to consider subdivisions of complexes more general than simplicial subdivisions of simplicial complexes. First we consider a very general situation in which the notion of "subdivision" is purely formal and devoid of geometric content, but for which an interesting theory nonetheless exists. In particular, the theory of Kazhdan-Lusztig polynomials occurs as a special case (see Example 6.9), though we add nothing new to the theory here. We also give a formal " $q$-analogue" of subdivisions of a simplex (see $\S 9$ ), though our results show that a rich geometric theory is unlikely. In $\S 7$ we specialize our general theory to a more geometric setting.

A poset $P$ is said to be locally finite if every (closed) interval $[x, y]$ is finite. We denote by $\hat{0}$ the unique element (when it exists) of $P$ satisfying $\hat{0} \leq t$ for all $t \in P$. A locally finite poset with $\hat{0}$ is graded if for all $t \in P$, every maximal chain of the interval $[\hat{0}, t]$ has the same length. We denote this length by $\rho(t)$, and call $\rho$ the rank function of $P$. A locally finite graded poset with $\hat{0}$ will be called lower graded.

A poset $P$ is called locally graded if $P$ is locally finite and for all $s \leq t$ in $P$, every maximal chain of the interval $[s, t]$ has the same length. We denote this length by $\rho(s, t)$. Thus $\rho(t, t)=0$, and if $s \leq u \leq t$ then $\rho(s, t)=\rho(s, u)+\rho(u, t)$. If $P$ is locally graded with $\hat{0}$ then $P$ is (lower) graded, and we have $\rho(s, t)=\rho(t)-\rho(s)$.

Fix a field $K$, and suppose that $P$ is locally finite. Let $I(P)$ denote the incidence algebra of $P$ (as defined e.g. in [ $\mathrm{Sta}_{11}$, Definition 3.6.1]) over the polynomial ring $K[x]$. As a $K$-vector space (or $K[x]$-module) $I(P)$ consists of all functions $f: \operatorname{Int}(P) \rightarrow K[x]$, where $\operatorname{Int}(P)$ denotes the set of (nonempty) intervals of $P$. The value of $f$ at the interval $[s, t]$ is denoted $f_{s t}=f_{s t}(x)$. We denote the convolution product in $I(P)$ simply by juxtaposition. Recall [ $\mathrm{Sta}_{11}$, Proposition 3.6.2] that $I(P)$ has a multiplicative identity (denoted either 1 or $\delta$ ), and that $f \in I(P)$ is invertible if and only if $f_{t t} \in K^{*}=K-\{0\}$ for all $t \in P$. The multiplicative identity $\delta$ of $I(P)$ is just the Kronecker delta. Let $K[x]^{P}$ denote the space of all functions $f: P \rightarrow K[x]$. We denote the value of $f \in K[x]^{P}$ at $t \in P$ by $f_{t}=f_{t}(x)$. If $P$ has a $\hat{0}$ (more generally, if every principal order ideal $\Lambda_{t}=\{s \in P: s \leq t\}$ is finite) then $I(P)$ acts on the right on the space $K[x]^{P}$ by the rule

$$
(f g)_{t}=\sum_{s \leq t} f_{s} g_{s t}, \quad g \in I(P) .
$$

Suppose $P$ is lower graded. For any $f \in K[x]^{P}$ satisfying $\operatorname{deg} f_{t} \leq \rho(t)$ for all $t \in P$, define $\bar{f}: P \rightarrow K[x]$ by

$$
\bar{f}_{t}(x)=x^{\rho(t)} f_{t}(1 / x)
$$


Note that $\overline{\bar{f}}=f$. Similarly if $P$ is locally graded, write

$$
I^{\prime}(P)=\left\{f \in I(P): \operatorname{deg} f_{s t} \leq \rho(s, t) \text { for all } s \leq t \text { in } P\right\} .
$$

It is easy to see that $I^{\prime}(P)$ is a subalgebra of $I(P)$. If $f \in I^{\prime}(P)$ then define $\bar{f} \in I^{\prime}(P)$ by

$$
\bar{f}_{s t}(x)=x^{\rho(s, t)} f_{s t}(1 / x) .
$$

Thus - is an involution on $I^{\prime}(P)$. A function $f \in K[x]^{P}$ or $f \in I^{\prime}(P)$ satisfying $f=\bar{f}$ is called symmetric.

6.1. Lemma. Suppose $f, g \in I^{\prime}(P)$. Then $\overline{f g}=\bar{f} \bar{g}$. If moreover $f$ is invertible, then $\bar{f}^{-1}=\overline{f^{-1}}$.

Proof. Let $s \leq t$ in $P$. We have

$$
(f g)_{s t}(x)=\sum_{s \leq u \leq t} f_{s u}(x) g_{u t}(x)
$$

Substitute $1 / x$ for $x$ and multiply by $x^{\rho(s, t)}$ to obtain $\overline{f g}=\bar{f} \bar{g}$. A similar argument applied to

$$
\delta_{s t}=\sum_{s \leq u \leq t} f_{s u}(x) f_{u t}^{-1}(x)
$$

shows $\bar{f}^{-1}=\overline{f^{-1}}$.

Define a function $\kappa \in I(P)$ to be unitary if $\kappa_{t t}=1$ for all $t \in P$. We now come to the key definitions of this section. They were suggested originally by the definition of "acceptable" in [ $\mathrm{Sta}_{10}$, p. 200].

6.2. Definition. (a) Let $P$ be a lower graded poset, and let $\kappa \in I(P)$ be unitary. A function $f \in K[x]^{P}$ is called $\kappa$-acceptable if $f \kappa=\bar{f}$, i.e.,

$$
\sum_{s \leq t} f_{s}(x) \kappa_{s t}(x)=\bar{f}_{t}(x),
$$

for all $t \in P$.

(b) Let $P$ be a locally graded poset, and let $\kappa \in I(P)$ be unitary. A function $f \in I(P)$ is called $\kappa$-totally acceptable if $f \kappa=\bar{f}$, i.e.,

$$
\sum_{s \leq u \leq t} f_{s u}(x) \kappa_{u t}(x)=\bar{f}_{s t}(x),
$$

for all $s \leq t$ in $P$. If there exists an invertible $\kappa$-totally acceptable function, then we call $\kappa$ a P-kernel.

Note that for fixed $\kappa$ the conditions of being $\kappa$-acceptable and $\kappa$-totally acceptable are linear; i.e., the functions satisfying them form a $K$-vector space (a subspace of $K[x]^{P}$ and $I(P)$ (or $I^{\prime}(P)$ ), respectively).

6.3. Proposition. Let $P$ be locally graded, and let $f \in I^{\prime}(P)$ be invertible. Then there exists a unique $\kappa \in I(P)$ (necessarily unitary) for which $f$ is $\kappa$-totally acceptable. 
Proof. Equation (22) states that $f \kappa=\bar{f}$ in $I(P)$, whence $\kappa=f^{-1} \bar{f}$.

6.4. Lemma (the generalized Dehn-Sommerville equations for the kernel $\kappa$ ). Let $Q$ be a finite graded poset with $\hat{0}$ and $\hat{1}$. Let $\kappa \in I^{\prime}(Q)$ be unitary and satisfy $\bar{\kappa}=\kappa^{-1}$. Finally let $f: Q-\{\hat{1}\} \rightarrow K[x]$ satisfy, for all $s<\hat{1}$,

$$
\sum_{u \leq s} f_{u}(x) \kappa_{u s}(x)=\bar{f}_{s}(x)
$$

(i.e., $f \kappa=\bar{f}$ on $Q-\{\hat{1}\})$. Then the polynomial

$$
g(x)=\sum_{s<\hat{1}} f_{s}(x) \kappa_{s \hat{1}}(x)
$$

is antisymmetric, i.e., $\bar{g}=-g$.

Proof. We have

$$
\begin{aligned}
\bar{g}(x) & =\sum_{s<\hat{1}} \bar{f}_{s}(x) \bar{\kappa}_{s \hat{1}}(x)=\sum_{s<\hat{1}}\left(\sum_{u \leq s} f_{u}(x) \kappa_{u s}(x)\right) \kappa_{s \hat{1}}^{-1}(x) \\
& =\sum_{u<\hat{1}} f_{u}(x) \sum_{u \leq s<\hat{1}} \kappa_{u s}(x) \kappa_{s \hat{1}}^{-1}(x)=\sum_{u<\hat{1}} f_{u}(x)\left(-\kappa_{u \hat{1}}(x) \kappa_{\hat{1} \hat{1}}^{-1}(x)\right) \\
& =-\sum_{u<\hat{1}} f_{u}(x) \kappa_{u \hat{1}}(x)=-g(x) .
\end{aligned}
$$

Note. We have called Lemma 6.4 the "generalized Dehn-Sommerville equations" for the following reason. Suppose $Q$ is Eulerian of rank $d+1$ and $\kappa_{s t}(x)=(x-1)^{\rho(t)-\rho(s)}$ for all $s \leq t$ in $Q$ (see $\S 7$, in particular Proposition 7.1), and let $g(x)$ be defined by (23). Then the polynomial $g(x) /(x-1)$ is the h-polynomial of $Q-\{\hat{1}\}$, as defined in [ $\left.\mathrm{Sta}_{10}, \S 2\right]$ or Example 7.2 below (more precisely, the vector of coefficients of $g(x) /(x-1)$ is called the $h$-vector of $Q-\{\hat{1}\})$. The generalized Dehn-Sommerville equations of [ $\mathrm{Sta}_{10}$, Theorem 2.4] assert that $g(x) /(x-1)$ is symmetric (as a polynomial of degree $d$ ). Clearly this is equivalent to $g(x)$ being antisymmetric (as a polynomial of degree $d+1$ ), as asserted by Lemma 6.4. If we take $Q-\{\hat{1}\}$ also to be simplicial, i.e., every interval $[0, t]$ is a boolean algebra, then we obtain the classical Dehn-Sommerville equations, essentially in the generality first given by Klee [Kl]. An additional reference to the generalized Dehn-Sommerville equations for Eulerian posets is [ $\mathrm{Sta}_{11}$, Theorem 3.14.9].

6.5. Theorem. Let $P$ be locally graded, and let $\kappa \in I(P)$ be unitary. The following two conditions are equivalent:

(a) $\kappa$ is a P-kernel, i.e, there exists an invertible $\kappa$-totally acceptable function $f \in I^{\prime}(P)$.

(b) $\bar{\kappa}=\kappa^{-1}$.

Proof. (a) $\Rightarrow$ (b). Given (a), we have $\kappa=f^{-1} \bar{f}$, from which it is immediate (using Lemma 6.1) that $\bar{\kappa}=\kappa^{-1}$. 
(b) $\Rightarrow$ (a) Assume (b). Let $\delta$ denote the identity element of $I(P)$ (so $\delta_{s t}$ is the Kronecker delta). For $s \leq t$ in $P$, define

$$
f_{s t}=\sum_{i \geq 0}\left(\begin{array}{c}
-1 / 2 \\
i
\end{array}\right)(\kappa-\delta)_{s t}^{i},
$$

where $(\kappa-\delta)_{s t}^{i}$ denotes the value of the function $(\kappa-\delta)^{i} \in I(P)$ at the interval $[s, t]$. Since $\kappa_{t t}(x)=1$ for all $t \in P$, the right-hand side of (24) is actually a finite sum and hence is well-defined. Note that $f_{t t}(x)=1$ for all $t \in P$, so $f$ is invertible. By the binomial theorem we then have $f=\kappa^{-1 / 2}$ (i.e., $f^{2}=\kappa^{-1}$ ). Moreover, applying the involution ${ }^{-}$to (24) and using $\bar{\kappa}=\kappa^{-1}$ shows that $\bar{f}=f^{-1}$. From this it follow that $f \kappa=\bar{f}$, so $f$ is $\kappa$-totally acceptable.

6.6. Proposition. (a) Let $P$ be lower graded, and let $\kappa \in I(P)$ be a P-kernel. For any $t \in P$, let $\mathscr{A}_{t}(P)$ denote the space of all $\kappa$-acceptable functions restricted to the interval $[\hat{0}, t]$. Then

$$
\operatorname{dim} \mathscr{A}_{t}(P)=\sum_{s \leq t}\left\lfloor\frac{1}{2} \rho(s)+1\right\rfloor .
$$

Moreover, if $P$ is finite and $\mathscr{A}(P)$ denotes the space of all $\kappa$-acceptable functions, then

$$
\operatorname{dim} \mathscr{A}(P)=\sum_{s \in P}\left\lfloor\frac{1}{2} \rho(s)+1\right\rfloor .
$$

(b) Let $P$ be locally graded, and let $\kappa \in I(P)$ be a P-kernel. For any $s \leq t$ in $P$, let $\mathscr{T}_{\text {st }}(P)$ denote the space $\mathscr{T}(P)$ of all $\kappa$-totally acceptable functions restricted to the set $\operatorname{Int}([s, t])$ of intervals contained in $[s, t]$. Then

$$
\operatorname{dim} \mathscr{T}_{s t}(P)=\sum_{s \leq u \leq v \leq t}\left\lfloor\frac{1}{2} \rho(u, v)+1\right\rfloor .
$$

Moreover, if $P$ is finite then

$$
\operatorname{dim} \mathscr{T}(P)=\sum_{u \leq v}\left\lfloor\frac{1}{2} \rho(u, v)+1\right\rfloor .
$$

Proof. (a) Let $t \in P$. Suppose that we have defined $f_{s}$ for all $s<t$, so that $(f \kappa)_{s}=\bar{f}_{s}$. We will show that the set of all polynomials $f_{t}$ satisfying $(f \kappa)_{t}=\vec{f}_{t}$ is a vector space of dimension $\left\lfloor\frac{1}{2} \rho(t)+1\right\rfloor$, from which $(25)$ and (26) follow.

Let $m=\rho(t)$. The condition $(f \kappa)_{t}=\bar{f}_{t}$ can be rewritten as

$$
\sum_{s<t} f_{s} \kappa_{s t}=\bar{f}_{t}-f_{t}
$$

By Lemma 6.4, the left-hand side of (27) is antisymmetric. Hence we may arbitrarily choose the coefficients $\alpha_{i}$ of $x^{i}$ in $f_{t}$ for $0 \leq i \leq\lfloor m / 2\rfloor$ (or for $\lceil m / 2\rceil \leq i \leq m)$, and this uniquely determines all of a polynomial $f_{t}$ satisfying (27). Thus we have $\lfloor m / 2\rfloor+1$ degrees of freedom in choosing $f_{t}$, as desired. 
(b) This follows from (a) and the fact that $f \in I([s, t])$ is $\kappa$-totally acceptable if and only if for all $u \in[s, t]$, the function $g \in K[x]^{[u, t]}$ defined by $g_{v}=f_{u v}$ is $\kappa$-acceptable.

6.7. Corollary. (a) Let $P$ be a lower graded poset, and let $\kappa$ be a P-kernel. Then there exists a unique $\kappa$-acceptable function $\gamma(P, \kappa)=\gamma: P \rightarrow K[x]$ satisfying

$$
\gamma_{\hat{0}}(x)=1, \quad \operatorname{deg} \gamma_{t}(x) \leq\left\lfloor\frac{1}{2}(\rho(t)-1)\right\rfloor, \quad t>\hat{0} .
$$

(b) Similarly if $P$ is locally graded and $\kappa$ is a P-kernel, then there is a unique $\kappa$-totally acceptable function $\gamma(P, \kappa)=\gamma \in I(P)$ satisfying

$$
\begin{gathered}
\gamma_{t t}(x)=1, \quad \text { for all } t \in P, \\
\operatorname{deg} \gamma_{s t}(x) \leq\left\lfloor\frac{1}{2}(\rho(s, t)-1)\right\rfloor, \quad \text { for all } s \leq t \text { in } P .
\end{gathered}
$$

Proof. (a) According to the proof of Proposition 6.6, once we have chosen $f_{s}(x)$ for all $s<t$ (where $\rho(t)=m>0$ ), we uniquely determine $f_{t}(x)=\alpha_{0}+$ $\alpha_{1} x+\cdots+\alpha_{m} x^{m}$ by choosing $\alpha_{m}, \alpha_{m-1}, \ldots, \alpha_{\lceil m / 2\rceil}$. Hence we must choose $\alpha_{m}=\alpha_{m-1}=\cdots=\alpha_{\lceil m / 2\rceil}=0$ to insure that $\operatorname{deg} f_{t} \leq\left\lfloor\frac{1}{2}(m-1)\right\rfloor$, so $\gamma$ exists and is unique.

(b) This follows from (a) for the same reason that Proposition 6.6(b) follows from Proposition 6.6(a).

Note that the proof of Proposition 6.5, together with Corollary 6.7(b), gives two "canonical" examples of $\kappa$-totally acceptable functions on a locally graded point $P$ with $P$-kernel $\kappa$, viz., $\kappa^{-1 / 2}$ and $\gamma(P, \kappa)$. As will become apparent from Example 6.9 and much of $\S 7$, the function $\gamma(P, \kappa)$ is in general the more interesting and important of the two.

6.8. Example. Let $P$ be a locally graded poset. Perhaps the simplest nontrivial example of a kernel $\kappa=f^{-1} \bar{f}$ is obtained by choosing $f=\zeta$ (the zeta function of $P$, defined by $\zeta_{s t}=1$ for all $s \leq t$ in $P$ ). Then

$$
\kappa_{s t}(x)=\sum_{s \leq u \leq t} \mu_{s u} x^{\rho(u, t)},
$$

where $\mu=\zeta^{-1}$ is the Möbius function of $P$ [ $\operatorname{Sta}_{11}$, Chapter 3.7]. Thus $\kappa_{s t}(x)$ is the characteristic polynomial $\chi_{s t}(x)$ of the interval $[s, t]$ (see [ $\mathrm{Sta}_{11}, \mathrm{pp}$. 128-129]). Of course for this $P$-kernel $\chi$ we have $\gamma(P, \chi)=\zeta$.

6.9. Example. One of the deepest and most interesting examples of a $P$-kernel arises from the Kazhdan-Lusztig theory of representations of Hecke algebras [K-L; Hu, Chapter 7]. We adhere to the terminology and notation of [K-L]. Let $W$ be a (not necessarily finite) Coxeter group, and let $S$ be the corresponding set of simple reflections. Regard $W$ as a poset endowed with the Bruhat order. 
Define a function $R \in I(W)$ inductively as follows:

$$
\begin{aligned}
& R_{u u}=1, \quad \text { for all } u \in W \\
& R_{u v}= \begin{cases}R_{s u, s v}, & \text { if } s u<u \text { and } s v<v(\text { for some } s \in S), \\
R_{u s, v s}, & \text { if } u s<u \text { and } v<v, \\
(x-1) R_{s u, v}+x R_{s u, s v}, & \text { if } s u>u \text { and } s v<v .\end{cases}
\end{aligned}
$$

(For an explicit but complicated formula for $R$, see [D].) Then [K-L, Lemma $2.1(\mathrm{i}, \mathrm{ii})]$ implies that $R$ is a $W$-kernel. From [K-L, (2.2.6)] and the definition of $P_{u v}$, it follows that the function $\gamma=\gamma(W, R)$ is given by

$$
\gamma_{u v}(x)=(-1)^{\rho(u, v)} P_{u v}(x),
$$

where $P_{u v}$ is the Kazhdan-Lusztig polynomial. (Note. The involution - in [KL] is not quite the same as our ${ }^{-}$.) The $W$-kernel $R$ has the special property [K-L, Lemma 2.1(i)]

$$
\bar{R}_{u v}=(-1)^{\rho(u, v)} R_{u v} ;
$$

i.e., $R_{u v}$ is symmetric (respectively, antisymmetric) for $\rho(u, v)$ even (respectively, odd). The function $\bar{R}$ is also a $W$-kernel, and for this $W$-kernel we have simply $\gamma(W, \bar{R})=P$.

We now come to a very general symmetry result. In the next section (after the proof of Corollary 7.7) we will explain how it includes Theorem 3.3 as a special case.

6.10. Proposition. Let $P$ be lower graded (respectively, locally graded) with $P$ kernel $\kappa$. Let $g \in I^{\prime}(P)$ be $\kappa$-totally acceptable and invertible. Then $f \in K[x]^{P}$ (respectively, $\left.f \in I^{\prime}(P)\right)$ is $\kappa$-acceptable (respectively, $\kappa$-totally acceptable) if and only if $\mathrm{fg}^{-1}$ is symmetric.

Proof. We have (using Lemma 6.1 and Theorem 6.5 when appropriate)

$$
f g^{-1}=\overline{f g^{-1}} \Longleftrightarrow f\left(\bar{g} \kappa^{-1}\right)^{-1}=\overline{f g^{-1}} \Longleftrightarrow f \kappa=\bar{f} \text {. }
$$

Proposition 6.10 shows that every $\kappa$-totally acceptable function has the form $\kappa^{-1 / 2}(f+\bar{f})$ for $f \in I^{\prime}(P)$ (or equivalently, setting $f=\kappa^{1 / 2} g, g+\kappa^{-1} \bar{g}$ for $\left.g \in I^{\prime}(P)\right)$, and conversely every such function is totally acceptable. In particular, for any $\alpha \in K$ we have that $k_{\alpha}:=\kappa^{-1 / 2}\left(\kappa^{\alpha}+\kappa^{-\alpha}\right)$ is a $\kappa$-totally acceptable function commuting with $f$. This leads to several questions which we leave to the reader to investigate: (a) Let $\mathscr{V}$ be the vector space spanned by all $k_{\alpha}$ with $\alpha \in K$. What is the dimension of $\mathscr{V}$ ? Which $k_{\alpha}$ 's form a basis for $\mathscr{V}$ ? (b) Does $\mathscr{V}$ consist of all $\kappa$-totally acceptable functions which commute with $\kappa$ ? If not, what further ones are needed?

We conclude this section with a result on the structure of the set $\mathscr{K}(P)$ of all $P$-kernels. 
6.11. Proposition. Let $P$ be locally graded. Let $\tilde{I}(P)$ be the multiplicative group of all unitary $f \in I^{\prime}(P)$. Let

$$
\tilde{S}(P)=\{f \in \tilde{I}(P): f=\bar{f}\},
$$

so $\tilde{S}(P)$ is a subgroup of $\tilde{I}(P)$. Let $\tilde{I}(P) / \tilde{S}(P)$ be the set of all right cosets $\tilde{S}(P) \cdot f, f \in \tilde{I}(P)$, and let $\mathscr{K}(P)$ be the set of all P-kernels. Finally define

$$
G(P)=\left\{f \in \tilde{I}(P): \operatorname{deg} f_{s t} \leq\left\lfloor\frac{1}{2}(\rho(s, t)-1)\right\rfloor \text { for all } s<t \text { in } P\right\} .
$$

Thus $G(P)$ is an affine subspace (over $K)$ of $I^{\prime}(P)$. Define maps $\varphi_{1}: \tilde{I}(P) / \tilde{S}(P)$ $\rightarrow \mathscr{K}(P)$ and $\varphi_{2}: G(P) \rightarrow \mathscr{K}(P)$ by $\varphi_{1}(\tilde{S}(P) \cdot f)=f^{-1} \bar{f}$ and $\varphi_{2}(f)=f^{-1} \bar{f}$. Then $\varphi_{1}$ and $\varphi_{2}$ are bijections. In fact, $G(P)$ forms a set of right coset representatives of $\tilde{S}(P)$ in $\tilde{I}(P)$, and $\varphi_{1}^{-1}(\kappa)=\tilde{S}(P) \cdot \kappa^{-1 / 2}, \varphi_{2}^{-1}(\kappa)=\gamma(P, \kappa)$.

Proof. If $\tilde{S}(P) \cdot f=\tilde{S}(P) \cdot g$, then $f=p g$ for some $p=\bar{p}$, so $f^{-1} \bar{f}=$ $(p g)^{-1}(\overline{p g})=g^{-1} \bar{g}$. Hence $\varphi_{1}$ is well defined. By the proof of Proposition 6.3, $\varphi_{1}$ is surjective. By Proposition 6.10, $\varphi_{1}$ is injective. The remaining statements follow from Corollary 6.7(b) and simple direct arguments.

\section{EULERIAN POSETS AND THEIR SUBDIVISIONS}

In this section we deal with a certain $P$-kernel $\lambda$ which will allow us to extend many of our results on local $h$-vectors to more general types of subdivisions. For any locally graded poset $P$, define a function $\lambda \in I^{\prime}(P)$ by

$$
\lambda_{s t}(x)=(x-1)^{\rho(s, t)},
$$

for all $s \leq t$ in $P$. Recall that a finite poset $P$ is Eulerian [ $\mathrm{Sta}_{11}$, Chapter 3.14] if it is graded, has a $\hat{0}$ and $\hat{1}$, and its Möbius function $\mu$ is given by

$$
\mu(s, t)=(-1)^{\rho(s, t)}
$$

for all $s \leq t$ in $P$. (Equivalently, every interval $[s, t]$ with $s<t$ has as many elements of even rank as of odd rank.) A lower graded (respectively, locally graded) poset $P$ is lower Eulerian (respectively, locally Eulerian) if every interval is Eulerian.

7.1. Proposition. Let $P$ be locally graded. Then $\lambda$ is a P-kernel if and only if $P$ is locally Eulerian.

Proof. Let $P$ be locally graded, and let $s \leq t$ in $P$. Then

$$
\begin{aligned}
(\bar{\lambda} \lambda)_{s t} & =\sum_{s \leq u \leq t} x^{\rho(s, u)}\left(\frac{1}{x}-1\right)^{\rho(s, u)}(x-1)^{\rho(u, t)} \\
& =(x-1)^{\rho(s, t)} \sum_{s \leq u \leq t}(-1)^{\rho(s, u)} .
\end{aligned}
$$


Hence $\bar{\lambda}=\lambda^{-1}$ (i.e., $\lambda$ is a $P$-kernel) if and only if

$$
\delta_{s t}=\sum_{s \leq u \leq t}(-1)^{\rho(s, u)}
$$

for all $s \leq t$ in $P$. But by the defining recurrence [ $\operatorname{Sta}_{11}$, Chapter 3.7, (14)] for $\mu$, equation (28) is equivalent to $\mu_{s t}=(-1)^{\rho(s, t)}$ for all $s \leq t$ in $P$.

For the remainder of this section we will say simply acceptable and totally acceptable for $\lambda$-acceptable and $\lambda$-totally acceptable. Moreover, $\gamma$ will always denote $\gamma(P, \lambda)$.

7.2. Example. Let $P$ be lower Eulerian. The polynomial $\gamma_{t}(x)$ is denoted by $g\left(P_{t}, x\right)$ in $\left[\operatorname{Sta}_{10}\right]$. If $P$ is Eulerian and $\gamma_{\hat{1}}(x)=h_{0}+h_{1} x+\cdots+h_{s} x^{s}$ (where we can take $s=\left\lfloor\frac{1}{2}(\rho(\hat{1})-1)\right\rfloor$, then $\left(h_{0}, \ldots, h_{s}\right)$ is called in $\left[\mathrm{Sta}_{10}, \S 2\right]$ the (generalized) h-vector of $P$ or $g$-vector of $P-\{\hat{1}\}$.

When $P$ is the boolean algebra $B_{d}$ of rank $d$, we have $\gamma_{t}(x)=1$ for all $t \in B_{d}\left[\mathrm{Sta}_{10}\right.$, Proposition 2.1; $\mathrm{Sta}_{11}$, Example 3.14.8]. For an arbitrary finite lower Eulerian poset $P$ of rank $d$, the $h$-vector $h(P)=\left(h_{0}, \ldots, h_{d}\right)$ is defined by

$$
\bar{h}(P, x)=h_{0} x^{d}+h_{1} x^{d-1}+\cdots+h_{d}=\sum_{t \in P} \gamma_{t}(x)(x-1)^{d-\rho(t)}
$$

When $P$ is Eulerian, (29) is consistent with our definition

$$
h(P, x)=h_{0}+h_{1} x+\cdots+h_{d} x^{d}=\gamma_{\hat{1}}(x),
$$

since $\gamma$ is acceptable.

We now turn to some examples of acceptable and totally acceptable functions on lower or locally Eulerian posets. Our first result is included for the sake of completeness, though we do not know of any reasonable application. First note that for $P$ locally Eulerian, it follows from Proposition 7.1 and the proof of Proposition 6.5 that $\lambda^{-1 / 2}$ is totally acceptable. For any $\alpha \in K$, a simple argument shows

$$
\lambda_{s t}^{\alpha}(x)=\zeta_{s t}^{\alpha} \lambda_{s t}(x)=\zeta_{s t}^{\alpha}(x-1)^{\rho(s, t)}
$$

for all $s \leq t$ in $P$. (For any unitary $f \in I(P)$ we define, analogously to (24),

$$
\left.f_{s t}^{\alpha}=\sum_{n \geq 0}\left(\begin{array}{l}
\alpha \\
n
\end{array}\right)(f-\delta)_{s t}^{n} .\right)
$$

In particular, for the boolean algebra $B_{d}$ one can compute $\zeta_{s t}^{\alpha}=\alpha^{\rho(s, t)}$, so $\lambda_{s t}^{-1 / 2}=\left(\frac{1}{2}(1-x)\right)^{\rho(s, t)}$ (see [ $\mathrm{Sta}_{11}$, Example 3.11.2]). Equation (30) in the case $\alpha=-1 / 2$ motivates the following result. The proof is straightforward and will be omitted. 
7.3. Proposition. (a) Let $P$ be lower Eulerian. Given $a: P \rightarrow K$, define $\hat{a}: P \rightarrow K[x] b y$

$$
\hat{a}_{t}(x)=a_{t}(x-1)^{\rho(t)} .
$$

Then $\hat{a}$ is acceptable if and only if

$$
(a \zeta)_{t}=(-1)^{\rho(t)} a_{t}
$$

for all $t \in P$. The set of all such a forms a vector space over $K$ of dimension $\#\{t \in P: \rho(t)$ is even $\}$.

(b) Let $P$ be locally Eulerian. Given $a \in I(P, K)$ (the incidence algebra of $P$ over $K$ ), define $\hat{a} \in I(P)$ (the incidence algebra of $P$ over $K[x]$ ) by

$$
\hat{a}_{s t}(x)=a_{s t}(x-1)^{\rho(s, t)} .
$$

Then $\hat{a}$ is totally acceptable if and only if

$$
(a \zeta)_{s t}=(-1)^{\rho(s, t)} a_{s t},
$$

for all $s \leq t$ in $P$. The set of all such a forms a vector space over $K$ of

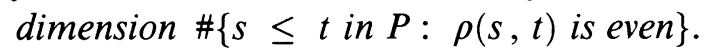

We now will give a more significant example of an acceptable function on a lower Eulerian poset. The key definition is the following.

7.4. Definition. Let $P$ be a lower Eulerian poset. A formal subdivision of $P$ consists of a lower Eulerian poset $\Gamma$ and a surjective function $\sigma: \Gamma \rightarrow P$ with the following properties:

(i) For all $t \in \Gamma, \rho(t) \leq \rho(\sigma(t))$.

(ii) For every $F \in P$, let $P_{\leq F}=\{G \in P: G \leq F\}$. Then $\Gamma_{F}:=\sigma^{-1}\left(P_{\leq F}\right)$ is an order ideal of $\Gamma$; i.e., if $t \in \Gamma_{F}$ and $s<t$, then $s \in \Gamma_{F}$. We think of $\Gamma_{F}$ as the "restriction" of $\Gamma$ to $F$.

(iii) Define the interior $\operatorname{int}\left(\Gamma_{F}\right)$ of the poset $\Gamma_{F}$ by $\operatorname{int}\left(\Gamma_{F}\right)=\sigma^{-1}(F)$. Let $h\left(\Gamma_{F}, x\right)$ denote the $h$-polynomial of the lower Eulerian poset $\Gamma_{F}$ (see (29)); i.e.,

$$
x^{\rho(F)} h\left(\Gamma_{F}, 1 / x\right)=\sum_{t \in \Gamma_{F}} \gamma_{t}(x)(x-1)^{\rho(F)-\rho(t)},
$$

or equivalently,

$$
h\left(\Gamma_{F}, x\right)=\sum_{t \in \Gamma_{F}} \bar{\gamma}_{t}(x)(-1)^{\rho(F)-\rho(t)}(x-1)^{\rho(F)-\rho(t)} .
$$

Similarly define $h\left(\operatorname{int}\left(\Gamma_{F}\right), x\right)$ by replacing $\Gamma_{F}$ with $\operatorname{int}\left(\Gamma_{F}\right)$ in the above formula. Then we require that $\bar{h}\left(\Gamma_{F}, x\right)=h\left(\operatorname{int}\left(\Gamma_{F}\right), x\right)$; i.e.,

$$
x^{\rho(F)} h\left(\Gamma_{F}, 1 / x\right)=h\left(\operatorname{int}\left(\Gamma_{F}\right), x\right)
$$

Sometimes by abuse of notation we will speak of $\Gamma$ as being a formal subdivision of $P$, the map $\sigma: \Gamma \rightarrow P$ being tacitly understood. 
7.5. Theorem. Let $\sigma: \Gamma \rightarrow P$ be a formal subdivision of the lower Eulerian poset $P$. Let $f: P \rightarrow K[x]$ be defined by $f_{F}(x)=h\left(\Gamma_{F}, x\right)$. Then $f$ is acceptable.

Proof. Fix $F \in P$. Then

$$
\begin{aligned}
& \sum_{G \leq F} f_{G}(x)(x-1)^{\rho(G, F)} \\
& \quad=\sum_{G \in F}(x-1)^{\rho(G, F)} \sum_{t \in \Gamma_{G}} \bar{\gamma}_{t}(x)(-1)^{\rho(G)-\rho(t)}(x-1)^{\rho(G)-\rho(t)} \\
& \quad=\sum_{t \in \Gamma_{F}} \sum_{F \geq G \geq \sigma(t)} \bar{\gamma}_{t}(x)(x-1)^{\rho(F)-\rho(t)}(-1)^{\rho(G)-\rho(t)} .
\end{aligned}
$$

Now since the interval $[\sigma(t), F]$ is Eulerian we have

$$
\sum_{F \geq G \geq \sigma(t)}(-1)^{\rho(G)}=\delta_{F, \sigma(t)}(-1)^{\rho(F)} .
$$

Hence

$$
\begin{aligned}
\sum_{G \leq F} f_{G}(x)(x-1)^{\rho(G, F)} & =\sum_{t \in \operatorname{int}\left(\Gamma_{F}\right)} \bar{\gamma}_{t}(x)(x-1)^{\rho(F)-\rho(t)}(-1)^{\rho(F)-\rho(t)} \\
& =h\left(\operatorname{int}\left(\Gamma_{F}\right), x\right) \\
& =\bar{h}\left(\Gamma_{F}, x\right)=\bar{f}_{F}(x)
\end{aligned}
$$

so $f$ is acceptable.

Note. We do not see a reasonable way of extending the notion of formal subdivision to posets $P$ with a more general $P$-kernel $\kappa$. The proof of Theorem 7.5 uses the special property of the kernel $\lambda$ that if $s \leq u \leq t$, then $\lambda_{s t}=\lambda_{s u} \lambda_{u t}$.

Note. A special case of Theorem 7.5 with basically the same proof as here appears in $\left[\mathrm{Ba}_{2}\right.$, Proposition 6].

Theorem 7.5 raises the problem of finding "nice" examples of formal subdivisions. Thus we will discuss how the usual topological notion of subdivision is related to formal subdivisions. Let $P$ be a $\mathrm{CW}$-poset, in the sense of Björner [Bj]. This means that $P$ is the face poset of a regular $\mathrm{CW}$-complex, also denoted $P$. Equivalently, $P$ has a $\hat{0}$ and the order complex $\Delta(\hat{0}, t)$ of every open interval $(\hat{0}, t)$ of $P$ is a triangulation of a sphere. There is an obvious topological notion of a subdivision $\Gamma$ (as a regular $\mathrm{CW}$-complex, i.e., $\Gamma$ is a $\mathrm{CW}$-poset) of a regular $\mathrm{CW}$-complex $P$. Namely, we have a function $\sigma: \Gamma \rightarrow P$ satisfying properties (i) and (ii) above of formal subdivisions, but (iii) is replaced by:

(iii $^{\prime}$ ) For every $F \in P$, the poset $\Gamma_{F}-\{\hat{0}\}=\sigma^{-1}\left(P_{\leq F}\right)-\{\hat{0}\}$ is topologically a ball; i.e., its order complex is a triangulation of a ball. Moreover,

$$
\operatorname{int}\left(\Gamma_{F}\right)=\left\{p \in \Gamma: p \notin \partial \Gamma_{F}\right\},
$$


where $\partial \Gamma_{F}$ is the order ideal (subcomplex) of $\Gamma$ generated by all codimension one faces of $\Gamma_{F}$ contained in exactly one facet (maximal face) of $\Gamma_{F}$.

Let us call such a subdivision CW-regular, to distinguish it from a regular subdivision in the sense of Definition 5.1.

7.6. Proposition. Let $\sigma: \Gamma \rightarrow P$ be a $C W$-regular subdivision of the $C W$-poset $P$. Then $\sigma$ is a formal subdivision, so the function $h\left(\Gamma_{F}, x\right)$ is acceptable on $P$.

Proof. Let $F \in P$. We may assume $F>\hat{0}$, so $\Gamma_{F}$ is a nonempty ball. The "reciprocity theorem" (31) is then the special case of [ $\mathrm{Sta}_{10}$, Lemma 6.2] where we take $P$ in [ $\mathrm{Sta}_{10}$, Lemma 6.2] to be $\Gamma_{F}$, and $I$ in [ $\mathrm{Sta}_{10}$, Lemma 6.2] to be $\partial \Gamma_{F}$. (In the statement of [ $\mathrm{Sta}_{10}$, Lemma 6.2], the possibility that $I=\partial \Gamma_{F}$ is not allowed, but actually the proof goes through in this case without change.)

Note that if $\Delta$ and $\Delta^{\prime}$ are both simplicial complexes (which we identify with their poset of faces), then a CW-regular subdivision $\sigma: \Delta^{\prime} \rightarrow \Delta$ is just a subdivision of $\Delta$ into $\Delta^{\prime}$ as discussed in $\S 2$.

There exist certain kinds of geometrically meaningful subdivisions of a CWposet more general than CW-regular subdivisions. For instance, rather than requiring that $\Gamma_{F}-\{\hat{0}\}$ be a ball, it would suffice that $\Gamma_{F}$ is a Cohen-Macaulay pseudomanifold with boundary, where the boundary is given by $\partial \Gamma_{F}=\Gamma_{F}-$ $\operatorname{int}\left(\Gamma_{F}\right)$. We will not discuss such generalizations any further here.

7.7. Corollary. Let $\Gamma$ be a formal subdivision of the Eulerian poset $P$. Assume $P$ has rank $d$, and let $\gamma=\gamma(P, \lambda)$. Define

$$
\ell_{P}(\Gamma, x)=\sum_{t \in P} h\left(\Gamma_{t}, x\right) \gamma_{t \hat{1}}^{-1}(x) .
$$

Then

$$
x^{d} \ell_{P}(\Gamma, 1 / x)=\ell_{P}(\Gamma, x) .
$$

Proof. Apply Proposition 6.10 to the case $f_{t}(x)=h\left(\Gamma_{t}, x\right)$ (which is acceptable by Theorem 7.5) and $g_{s t}(x)=\gamma_{s t}(x)$, which is totally acceptable by definition of $\gamma_{s t}(x)$ (and the result that $\gamma_{s t}(x)$ actually exists, i.e., Corollary 6.7).

When $P$ is the boolean algebra $2^{V}$ in Corollary 7.7, then $\gamma_{s t}(x)=1$ for all $s \leq t$ (as essentially noted in Example 7.2), and $\gamma_{s t}^{-1}(x)=(-1)^{\rho(s, t)}$. Hence if $\Gamma$ is a simplicial subdivision of $2^{V}$, then comparing (4) and (32) shows that

$$
\ell_{P}(\Gamma, x)=\ell_{V}(\Gamma, x),
$$

the local $h$-vector as investigated in $\S \S 2-4$. Therefore Corollary 7.7 specializes to Theorem 3.3, so Theorem 3.3 is proved.

Equation (32) is thus the "correct" definition of the local $h$-vector of arbitrary CW-regular subdivisions of arbitrary Eulerian CW-posets (or even formal subdivisions of Eulerian posets). Since $\ell=h \gamma^{-1}$ if and only if $h=\ell \gamma$, it is equivalent (as a definition valid for all $\mathrm{CW}$-regular subdivisions of all Eulerian 
CW-posets) to

$$
h(\Gamma, x)=\sum_{t \in P} \ell_{P_{\leq t}}\left(\Gamma_{t}, x\right) \gamma_{t \hat{1}}(x) .
$$

This explains why we defined $\ell_{V}(\Gamma, x)$ by (3) and not (4). Equation (32) raises the question, however, of whether any kind of formula exists for $\gamma_{s t}^{-1}(x)$. An answer, in the general context of any $P$-kernel $\kappa$, will be given in Proposition 8.1 .

Note. We do not see how to extend the definition (32) of $\ell_{P}(\Gamma, x)$ to formal subdivisions $\Gamma$ of arbitrary lower Eulerian posets, generalizing the definition of $\ell_{\Delta}\left(\Delta^{\prime}\right)$ given by equation $(20)$.

Note. Following $\left[\mathrm{Ba}_{2}\right]$, define a formal subdivision $\sigma: \Gamma \rightarrow P$ of an Eulerian poset $P$ to be shallow if for all $F \in \Gamma$ we have

$$
\rho(\sigma(F))<2\left(\rho(F)-\operatorname{deg} \gamma_{F}(x)\right) .
$$

As in $\left[\mathrm{Ba}_{2}\right.$, Theorem 4], if $\Gamma$ is shallow then $h(\Gamma, x)=h(P, x)$. Moreover, if $\Gamma$ is an arbitrary formal subdivision of the Eulerian poset $P$ such that for all $t \in P$ we have $\ell_{P_{\leq t}}\left(\Gamma_{t}, x\right) \geq 0$ and $h\left(P_{\geq t}, x\right) \geq 0$ (by Theorem 7.9 this condition holds if $P$ is the face lattice of a rational polytope $\mathscr{P}$ and $\Gamma$ is the face poset of a rational geometric polyhedral subdivision of $\mathscr{P}$ ), then it follows easily from Theorem 7.8 that the following three conditions are equivalent: (a) $\Gamma$ is shallow, (b) $h(\Gamma, x)=h(P, x)$, and (c) $\ell_{P_{\leq t}}\left(\Gamma_{t}, x\right)=0$ for all $t>$ $\hat{0}$ in $P$. Thus under appropriate conditions we have a characterization of shallow subdivisions in terms of local $h$-vectors. (We did not discuss shallow subdivisions in Part I since a shallow subdivision can have no new vertices, so no nontrivial simplicial subdivision of a simplex is shallow.)

As pointed out after the proof of Corollary 7.7, that result generalizes Theorem 3.3 from simplicial subdivisions of a simplex to $\mathrm{CW}$-regular subdivisions of a CW-poset. We can ask whether our other main results on local $h$-vectors have a similar generalization. There is little difficulty in generalizing Theorem 3.2 , but we are only able to extend Corollary 4.7 in a special case for which we can apply the intersection homology of toric varieties. Similarly the generalization of Theorem 5.2 requires intersection homology theory, but this was also the case for Theorem 5.2 itself.

7.8. Theorem. Let $P$ be a pure lower Eulerian poset of rank $d$, and let $\Gamma$ be a formal subdivision of $P$. Then

$$
h(\Gamma, x)=\sum_{t \in P} \ell_{P_{\leq t}}\left(\Gamma_{t}, x\right) h\left(P_{\geq t}, x\right),
$$

where $h$ is defined by (29). 
Proof. By equation (29), Lemma 6.1, and the purity of $P$, we have

$$
h\left(P_{\geq t}, x\right)=\sum_{u \geq t} \bar{\gamma}_{t u}(x)(1-x)^{d-\rho(u)} .
$$

Also by Corollary 7.7 we have

$$
\ell_{P_{\leq t}}\left(\Gamma_{t}, x\right)=\sum_{s \leq t} \bar{h}\left(\Gamma_{s}, x\right) \bar{\gamma}_{s t}^{-1}(x) .
$$

Hence

$$
\begin{aligned}
\sum_{t \in P} \ell_{P_{\leq t}}\left(\Gamma_{t}, x\right) h\left(P_{\geq t}, x\right) & =\sum_{s \leq t \leq u} \bar{h}\left(\Gamma_{s}, x\right) \bar{\gamma}_{s t}^{-1}(x) \bar{\gamma}_{t u}(x)(1-x)^{d-\rho(u)} \\
& =\sum_{s \leq u} \bar{h}\left(\Gamma_{s}, x\right) \delta_{s u}(1-x)^{d-\rho(u)} \\
& =\sum_{s} \bar{h}\left(\Gamma_{s}, x\right)(1-x)^{d-\rho(s)} \\
& =h(\Gamma, x),
\end{aligned}
$$

since $h$ is acceptable (Theorem 7.5).

7.9. Theorem. (a) Let $P$ be the face-lattice of a rational (i.e., the vertices have rational coordinates) convex $d$-polytope $\mathscr{P}$. Let $\Gamma$ be a rational polyhedral subdivision of $P$ (or of $\mathscr{P})$; i.e., $\Gamma$ can be realized in Euclidean space as a geometric subdivision of the rational convex polytope $\mathscr{P}$, such that the faces of $\Gamma$ are rational convex polytopes. Let $\ell_{p}(\Gamma, x)=\ell_{0}+\ell_{1} x+\cdots+\ell_{d} x^{d}$. Then $\ell_{i} \geq 0$ (and $\ell_{i}=\ell_{d-i}$ by Corollary 7.7).

(b) If moreover $\Gamma$ is a regular subdivision (in the sense of Definition 5.1), then $0 \leq \ell_{0} \leq \ell_{1} \leq \cdots \leq \ell_{\lfloor d / 2\rfloor}$.

Proof. Analogous to the proof of Theorem 5.2, using the following variation of [ $\mathrm{Sta}_{10}$, Theorem 3.1]: Let $Q$ be the face-lattice of a rational polyhedral decomposition $\Gamma^{\prime}$ of a rational convex polytope, and let $\Sigma$ be a rational fan with cross-section $\Gamma^{\prime}$, as in the proof of Theorem 5.2. Let $X_{\Sigma}$ be the complex toric variety corresponding to $\Sigma$, and let $I H$ denote complex intersection homology with compact supports. Then $\operatorname{dim} I H^{2 i}\left(X_{\Sigma}\right)=h_{i}(Q)$, while $\operatorname{dim} I H^{2 i+1}\left(X_{\Sigma}\right)=$ 0 .

7.10. Conjecture. Theorem 7.9(a) (and perhaps also (b)) remains true if $P$ is any Cohen-Macaulay Eulerian lattice and $\sigma: \Gamma \rightarrow P$ any formal subdivision of $P$ satisfying: (i) $\Gamma$ is a Cohen-Macaulay meet-semilattice, and (ii) $\sigma$ is quasi-geometric is the sense that if $t \in \Gamma$ and $s_{1}, \ldots, s_{k}$ are the atoms of $\Gamma$ lying below $t$, then $\sigma\left(s_{1}\right) \vee \cdots \vee \sigma\left(s_{k}\right)=\sigma(t)$.

7.11. Corollary. Preserve the notation and hypotheses of Theorem 7.9(a). Then

$$
h(\Gamma, x) \geq h(P, x) .
$$


In particular, if $\Gamma$ is any triangulation of $P$ (which will be combinatorially equivalent to a rational triangulation when, as we areassuming, $\mathscr{P}$ is rational), then the number $f_{d}(\Gamma)$ of facets of $\Gamma$ satisfies

$$
f_{d}(\Gamma) \geq h(P, 1) \text {. }
$$

Proof. Analogous to the proof of Theorem 4.10. By Theorem 7.9(a) the polynomials $\ell_{P_{\leq t}}\left(\Gamma_{t}, x\right)$ appearing in (34) have nonnegative coefficients. By [ $\mathrm{Sta}_{10}$, Theorem 3.1] we similarly have $h\left(P_{\geq t}, x\right) \geq 0$. The term of (34) indexed by $t=\hat{0}$ is just $h(P, x)$, so it follows from (34) that $h(\Gamma, x) \geq h(P, x)$.

For any $(d+1)$-dimensional simplicial complex $\Delta$ we have $h(\Delta, 1)=f_{d}(\Delta)$, so (37) follows from (36).

One case of Corollary 7.11 of particular interest is when $\mathscr{P}$ is a $d$-cube. Considerable research has been done on finding the minimum number of facets in a triangulation of the $d$-cube (see [Ha] and the references cited there). Unfortunately equation (37) yields nothing new; it states that $f_{d}(\Gamma) \geq \frac{1}{d+1}\left(\begin{array}{c}2 d \\ d\end{array}\right)$, while the best bound known at present is $f_{d}(\Gamma) \geq c^{d} d !^{1 / 2}$. Perhaps a more detailed study of (34) will yield an improvement over known results, but this seems quite difficult.

7.12. Corollary (the Upper Bound Conjecture for $h$-vectors of rational polytopes). Let $\mathscr{P}$ be a rational convex $d$-polytope with $n$ vertices, with boundary $\partial \mathscr{P}$. Let $P$ be the face poset of $\partial \mathscr{P}$, and let $h(P, x)=h_{0}+h_{1} x+\cdots+h_{d} x^{d}$. (Thus by $\left[\mathrm{Sta}_{10}\right.$, Theorem 2.4] or by Lemma 6.4 and the comments following it, we have $h_{i}=h_{d-i}$.) Then $0 \leq h_{i} \leq\left(\begin{array}{c}n-d+i-1 \\ i\end{array}\right)$.

Proof. Let $\Gamma$ be a triangulation of $\partial \mathscr{P}$ without new vertices. (Such triangulations are well known and easily seen to exist.) Consider the formula for $h(\Gamma, x)$ given by (34). In this formula we have $\ell_{P_{\leq t}}\left(\Gamma_{t}, x\right) \geq 0$ by Theorem 7.9(a). For any $t \in P$ the subposet $P_{\geq t}$ is the face poset of the boundary complex of some rational polytope $\mathscr{P}_{t}$, so by [ $\mathrm{Sta}_{10}$, Corollary 3.2] we have $h\left(P_{\geq t}, x\right) \geq 0$. Now by the Upper Bound Conjecture for spheres [ $\mathrm{Sta}_{2}$ ] (or even for simplicial polytopes, since we can choose $\Gamma$ to be polytopal) we have $h_{i}(\Gamma) \leq\left(\begin{array}{c}n-d+i-1 \\ i\end{array}\right)$. When $t=\hat{0}$ in (34) we just get the term $h(P, x)$, so $h_{i}(P) \leq\left(\begin{array}{c}n-d+i-1 \\ i\end{array}\right)$.

The classical Upper Bound Conjecture for polytopes [Mc] or spheres [ $\mathrm{Sta}_{2}$ ] has a simple combinatorial interpretation in terms of $f$-vectors. We know of no such interpretation, however, for Corollary 7.12. We may conjecture a generalization of Corollary 7.12 along the lines of Conjecture 7.10; in particular, Corollary 7.12 should remain true without the assumption that $\mathscr{P}$ is rational.

Example 7.2, Proposition 7.3, and Theorem 7.5 (together with its special case, Proposition 7.6) give some "natural" examples of acceptable functions. There is one further example worth mentioning.

7.13. Example. Let $L$ be a lattice in $\mathbf{R}^{N}$. Let $\Lambda$ be an $L$-polyhedral complex in $\mathbf{R}^{N}$, i.e., a (finite) set of convex polytopes in $\mathbf{R}^{N}$ with vertices in $L$, such 
that (i) if $\mathscr{P} \in \Lambda$ and $\mathscr{Q}$ is a face of $\mathscr{P}$, then $\mathscr{Q} \in \Lambda$, and (b) if $\mathscr{P}, \mathscr{P}^{\prime} \in \Lambda$ then $\mathscr{P} \cap \mathscr{P}^{\prime}$ is a face (possibly empty) of $\mathscr{P}$ and of $\mathscr{P}^{\prime}$. For instance, $\Lambda$ could be the set of faces of some $L$-polytope $\mathscr{P}$. (Even more general objects than $L$-polyhedral complexes could be considered, but for simplicity we do not deal with them here.) Given an integer $n>0$ and a $d$-dimensional $L$-polytope $\mathscr{P} \subset \mathbf{R}^{N}$ define

$$
i(\mathscr{P}, n)=\#(n \mathscr{P} \cap L),
$$

where $n \mathscr{P}=\{n \alpha: \alpha \in \mathscr{P}\}$. Also set $i(\mathscr{P}, 0)=1$. It is known that $i(\mathscr{P}, n)$ is a polynomial function of $n$ of degree $d$, called the Ehrhart polynomial of $\mathscr{P}$ (see [ $\mathrm{Sta}_{11}$, Corollary 4.6.28]). It follows that if we set

$$
\omega(\mathscr{P}, x)=(1-x)^{d+1} \sum_{n \geq 0} i(\mathscr{P}, n) x^{n},
$$

then $\omega(\mathscr{P}, x)$ is a polynomial in $x$ of degree $\leq d$. It is noted in [ $\mathrm{Sta}_{10}$, p. 201] (for the lattice $L=\mathbf{Z}^{N}$ ) that $\omega$ is an acceptable function on $\Lambda$. Now suppose $\Lambda$ is the face lattice of a $d$-dimensional $L$-simplex in $\mathbf{R}^{N}$. Since $\Lambda$ is isomorphic to the boolean algebra $B_{d+1}$, the zeta function $\zeta \in I(\Lambda)$ is totally acceptable (see Example 7.2). Hence the function $c=\omega \zeta^{-1}$ is symmetric (Proposition 6.10). Explicitly, we have (since $\zeta_{s t}^{-1}=(-1)^{\rho(s, t)}$ )

$$
c_{t}(x)=\sum_{s \in \Gamma} \omega(s, x)(-1)^{\rho(s, t)} .
$$

The polynomial $c_{t}(x)$ was earlier considered by Betke and McMullen [B-M]. (They use $C^{*}(T, t)$ for our $c_{t}(x)$, where their $T$ is our $t$, and their $t$ is our $x$.) They give a purely geometric definition of $c_{t}(x)$ from which it clearly has nonnegative coefficients. The symmetry of $c_{t}(x)$ is their Lemma $3(a)$, while the analogue of our equation (3) is their Lemma 3(b). For any $L$-polyhedral complex $\Gamma$ in $\mathbf{R}^{N}$, one can define

$$
i(\Gamma, n)=\#(n \cdot|\Gamma| \cap L), \quad i(\Gamma, 0)=1,
$$

as a straightforward generalization of $(38)$. (Now $i(\Gamma, n)$ is a polynomial for $n>0$ but perhaps not at 0 .) The polynomial

$$
\omega(\Gamma, x)=(1-x)^{1+\operatorname{dim} \Gamma} \sum_{n \geq 0} i(\Gamma, n) x^{n}
$$

is the "Ehrhart analogue" of the $h$-polynomial $h(\Gamma, x)$. Betke and McMullen [B-M, Theorem 1] establish also the Ehrhart analogue of our Theorem 3.2. (This analogue is a special case of our Theorem 7.8.) The paper [B-M] of Betke and McMullen provided some of the motivation for this paper.

The above discussion suggests that it might be interesting to investigate the function $c:=\omega \gamma^{-1}: \Gamma \rightarrow K[x]$, where $\Gamma$ is the face lattice of a convex $L$ polytope $\mathscr{P}, \omega$ is given by (39), and $\gamma=\gamma(P, \lambda)$. (When $\mathscr{P}$ is a simplex we have $\gamma=\zeta$ so $c$ is the same as defined above.) Specifically: 
7.14. Conjecture. Let $\Gamma$ be the face lattice of a convex $L$-polytope (or even a Cohen-Macaulay $L$-polyhedral complex). With notation as above, we have $\omega \gamma^{-1} \geq 0$; i.e., for all $s \leq t$ in $\Gamma$, the polynomial $\left(\omega \gamma^{-1}\right)_{s t}(x)$ has nonnegative coefficients.

In view of Theorem 5.2 and Conjecture 5.4, it is tempting to conjecture that in fact $\left(\omega \gamma^{-1}\right)_{s t}(x)$ is unimodal for all $s \leq t$ in $\Gamma$. But [ Sta $_{13}$, Example 3.4] implies easily that this stronger conjecture is false.

Note. As mentioned after the proof of Theorem 4.6, some results in [G-Z-K] are connected with our Example 7.13. In particular, their definition of the "Newton number" of an $L$-triangulation of an $L$-simplex is equivalent in our notation to $c_{\hat{1}}(1)$. Thus their definition of "thin" triangulation is equivalent to the condition $c_{\hat{1}}(1)=0$, or equivalently, since $c_{\hat{1}}(x)$ has nonnegative coefficients, to the condition $c_{\hat{1}}(x)=0$.

\section{Dual posets and duAl KeRnels}

In this section we investigate the relation between $P$-kernels and $P^{*}$-kernels, where $P^{*}$ denotes the dual poset to $P\left[\mathrm{Sta}_{11}\right.$, Chapter 3.2]. As an application, we give a simple proof of a conjecture of Kalai which was later proved by Kalai and independently by Klapper.

Let $P$ be a locally graded poset with dual $P^{*}$. If $f \in I(P)$, then define $f^{*} \in I\left(P^{*}\right)$ by $f_{t s}^{*}=f_{s t}$ for all $s \leq t$ in $P$. One easily checks that $(f g)^{*}=$ $g^{*} f^{*}$, and that $f \in I^{\prime}(P)$ if and only if $f^{*} \in I^{\prime}\left(P^{*}\right)$, and that then $(\bar{f})^{*}=\overline{f^{*}}$. Moreover, if $f$ is invertible then $\left(f^{-1}\right)^{*}=\left(f^{*}\right)^{-1}$. In particular, $f \kappa=\bar{f}$ if and only if $\left(f^{-1}\right)^{*}\left(\kappa^{-1}\right)^{*}=\overline{\left(f^{-1}\right)^{*}}$. Thus $\kappa$ is a $P$-kernel if and only if $\bar{\kappa}^{*}$ is a $P^{*}$-kernel, since $\bar{\kappa}=\kappa^{-1}$.

8.1. Proposition. Let $P$ be lower graded and $\kappa$ a P-kernel. Then

$$
\gamma\left(P^{*}, \bar{\kappa}^{*}\right)=\left(\gamma(P, \kappa)^{-1}\right)^{*}
$$

Proof. Write $\gamma=\gamma(P, \kappa)$. Thus $\gamma \kappa=\bar{\gamma}$. Applying ${ }^{-1}$ and ${ }^{*}$ (and using $\left.\kappa^{-1}=\bar{\kappa}\right)$ yields $\left(\gamma^{-1}\right)^{*} \bar{\kappa}^{*}=\overline{\left(\gamma^{-1}\right)^{*}}$. Hence $\left(\gamma^{-1}\right)^{*}$ is $\bar{\kappa}^{*}$-totally acceptable. Clearly $\left(\gamma^{-1}\right)_{t t}^{*}=1$ for all $t \in P^{*}$.

It remains to show

$$
\operatorname{deg}\left(\gamma^{-1}\right)_{t s}^{*} \leq\left\lfloor\frac{1}{2}\left(\rho^{*}(t, s)-1\right)\right\rfloor
$$

for all $t<s$ in $P^{*}$, where $\rho^{*}$ denotes the rank function of $P^{*}$. The proof is by induction on $\rho^{*}(t, s)$. When $\rho^{*}(t, s)=1$ we have $\left(\gamma^{-1}\right)_{t s}^{*}=-\gamma_{s t}$, so the assertion is true in that case. Now assume $\rho^{*}(t, s)>1$. We have

$$
0=\sum_{s \leq u \leq t} \gamma_{s u} \gamma_{u t}^{-1}
$$


For $s<u<t$ it follows by induction that

$$
\begin{aligned}
\operatorname{deg} \gamma_{s u} \gamma_{u t}^{-1} & \leq\left\lfloor\frac{1}{2}(\rho(s, u)-1)\right\rfloor+\left\lfloor\frac{1}{2}(\rho(u, t)-1)\right\rfloor \\
& \leq\left\lfloor\frac{1}{2}(\rho(s, t)-1)\right\rfloor .
\end{aligned}
$$

For $u=t$ we get

$$
\operatorname{deg} \gamma_{s t} \gamma_{t t}^{-1}=\operatorname{deg} \gamma_{s t} \leq\left\lfloor\frac{1}{2}(\rho(s, t)-1)\right\rfloor
$$

Hence from (41) the remaining term $\gamma_{s s} \gamma_{s t}^{-1}=\gamma_{s t}^{-1}$ also must satisfy $\operatorname{deg} \gamma_{s t}^{-1} \leq$ $\left\lfloor\frac{1}{2}(\rho(s, t)-1)\right\rfloor$, so the claim is proved.

We have shown that $\left(\gamma^{-1}\right)^{*}$ satisfies all the conditions which characterize $\gamma\left(P^{*}, \bar{\kappa}^{*}\right)$, so we must have $\left(\gamma^{-1}\right)^{*}=\gamma\left(P^{*}, \bar{\kappa}^{*}\right)$, as desired.

8.2. Lemma. Let $P$ be locally graded, and let $\kappa$ be a P-kernel. For $f \in I(P)$ and $\alpha \in K^{*}$ define $f_{\alpha} \in I(P)$ by

$$
\left(f_{\alpha}\right)_{s t}=\alpha^{\rho(s, t)} f_{s t} .
$$

Then $\kappa_{\alpha}$ is a P-kernel, and $\gamma\left(P, \kappa_{\alpha}\right)=\gamma(P, \kappa)_{\alpha}$.

Proof. Straightforward.

8.3. Corollary. Let $P$ be locally Eulerian, and let $\lambda^{*}$ denote the kernel $\lambda$ for the poset $P^{*}$; i.e.,

$$
\lambda_{t s}^{*}(x)=(x-1)^{\rho^{*}(t, s)}=(x-1)^{\rho(s, t)}=\lambda_{s t}(x),
$$

for all $s \leq t$ in $P$. Let $\gamma=\gamma(P, \lambda)$ and $\gamma^{*}=\gamma\left(P^{*}, \lambda^{*}\right)$. Then for all $s \leq t$ in $P$,

$$
\gamma_{t s}^{*}(x)=(-1)^{\rho(s, t)} \gamma_{s t}^{-1}(x)
$$

Proof. Note that $\bar{\lambda}=\lambda_{-1}$, in the notation of (42). Now use Proposition 8.1 and Lemma 8.2.

8.4. Corollary. Let $\Gamma$ be a formal subdivision of the Eulerian poset $P$. Then

$$
\ell_{P}(\Gamma, x)=\sum_{t \in P} h\left(\Gamma_{t}, x\right)(-1)^{\rho(t, \hat{1})} \gamma_{\hat{1} t}^{*}(x) .
$$

Proof. Immediate from Corollary 8.3 and (32).

Corollary 8.3 may be regarded as giving the relation between the $h$-vector of the Eulerian poset $P$ (as defined in Example 7.2) and of its dual $P^{*}$. As is well known, one cannot be computed solely in terms of the other (i.e., $\gamma_{\hat{0} \hat{1}}(x)$ does not determine $\left.\gamma_{\hat{1} \hat{0}}^{*}(x)\right)$. But Corollary 8.3 shows that knowing $\gamma_{s t}(x)$ for every $s \leq t$ in $P$ then determines $\gamma_{t s}^{*}(x)$ for every $t \leq s$ in $P^{*}$.

8.5. Corollary. Let $P$ be Eulerian of rank $d$ and $P-\{\hat{1}\}$ simplicial; i.e., the interval $[\hat{0}, t]$ is a boolean algebra for all $t<\hat{1}$ in $P$. If $\# P>1$, then

$$
\gamma_{\hat{0} \hat{1}}(x)+\sum_{t<\hat{1}}(-1)^{d-\rho(t)} \gamma_{1 t}^{*}(x)=0 .
$$


Proof. By Corollary 8.3,

$$
\sum_{t \in P} \gamma_{\hat{0} t}(x)(-1)^{d-\rho(t)} \gamma_{\hat{1} t}^{*}(x)=0 .
$$

Since $P-\{\hat{1}\}$ is simplicial, we have $\gamma_{\hat{0} t}(x)=1$ for all $t<\hat{1}$. Since trivially $\gamma_{\hat{1} \hat{1}}^{*}(x)=1$, the proof follows.

Corollary 8.5 yields a recursive method for computing the $h$-vector of an $\mathrm{Eu}$ lerian poset $P^{*}$ for which $P^{*}-\left\{\hat{0}^{*}\right\}$ is simple (the dual of simplicial). Although one can in fact compute $\gamma_{\hat{1} \hat{0}}(x)=h\left(P^{*}, x\right)$ just in terms of $\gamma_{\hat{0} \hat{1}}(x)=h(P, x)$ when $P-\{\hat{1}\}$ is simplicial (as follows from [ $\mathrm{Ba}_{1}$, Proposition 6.1]), and thus not requiring knowledge of each $\gamma_{\hat{1} t}^{*}(x)$ for $t>\hat{0}$, Corollary 8.5 has the virtues that (a) it is completely explicit, unlike what follows directly from [ $\left.\mathrm{Ba}_{1}\right]$, (b) it is simpler than the original recursive definition of $\gamma$, and (c) it is sometimes more convenient for applications. To illustrate this last point, we will obtain a generating function for the $h$-vector of the face lattice of the $d$-cube which was overlooked in our earlier work [ $\mathrm{Sta}_{10}$, Proposition 2.6] on this topic. Let $\gamma_{d}(x)=h\left(P_{d}, x\right)$, where $P_{d}$ is the face lattice of the $d$-cross-polytope (or $d$ octahedron) $K_{d}$. Since the $h$-polynomial of the boundary $\partial K_{d}$ of $K_{d}$ is well known to be $h\left(P_{d}-\{\hat{1}\}, x\right)=(1+x)^{d}$, we in fact have

$$
\gamma_{d}(x)=\sum_{i=0}^{\lfloor d / 2\rfloor}\left[\left(\begin{array}{c}
d \\
i
\end{array}\right)-\left(\begin{array}{c}
d \\
i-1
\end{array}\right)\right] x^{i}
$$

(where $\left.\left(\begin{array}{c}d \\ -1\end{array}\right)=0\right)$. Let $\gamma_{d}^{*}(x)=h\left(P_{d}^{*}, x\right)$, where $P_{d}^{*}$ is the dual of $P_{d}$, i.e., the face lattice of the $d$-cube. In the notation of $\left[\mathrm{Sta}_{10}\right.$, Proposition 2.6], we have $\gamma_{d}^{*}(x)=g\left(L_{d}, x\right)$.

8.6. Proposition. We have

$$
\sum_{d \geq 0} \frac{\gamma_{d}^{*}(x) y^{d}}{d !}=e^{2 y} \sum_{d \geq 0} \frac{(-1)^{d} \gamma_{d}(x) y^{d}}{d !}
$$

Proof. Apply (43) to $P=P_{d}$. Since the $d$-cube has $2^{d-i}\left(\begin{array}{c}d \\ i\end{array}\right) \quad i$-faces (which are $i$-cubes), we get

$$
\gamma_{d}(x)+\sum_{i=0}^{d}(-1)^{i+1} 2^{d-i}\left(\begin{array}{l}
d \\
i
\end{array}\right) \gamma_{i}^{*}(x)=0 .
$$

By standard generating function arguments this is equivalent to (44).

To conclude this section, we give a simple, conceptual proof of a generalization of a conjecture of $\mathrm{G}$. Kalai. The original conjecture of Kalai was proved independently by Kalai (unpublished) and A. Klapper (see [ $\mathrm{Ba}_{1}, \S 7$; B-K, Corollary to Theorem 8]) and is restated here as Corollary 8.8.

8.7. Proposition. Let $P$ be lower graded and $\kappa$ a P-kernel. Let $\gamma=\gamma(P, \kappa) \in$ $I(P)$ and $\tilde{\gamma}=\gamma\left(P^{*}, \bar{\kappa}^{*}\right)$. Fix $s<t$ such that $\rho(s, t)=2 e+1$ (an odd integer). 
Let

$$
\begin{aligned}
& \gamma_{s t}(x)=a_{0}+a_{1} x+\cdots+a_{e} x^{e} \\
& \tilde{\gamma}_{t s}(x)=a_{0}^{*}+a_{1}^{*} x+\cdots+a_{e}^{*} x^{e}
\end{aligned}
$$

Then $a_{e}=-a_{e}^{*}$.

Proof. By Proposition 8.1, we have

$$
\sum_{s \leq u \leq t} \gamma_{s u} \tilde{\gamma}_{t u}=0
$$

If $s<u<t$, then

$$
\operatorname{deg} \gamma_{s u} \tilde{\gamma}_{t u} \leq\left\lfloor\frac{1}{2}(\rho(s, u)-1)\right\rfloor+\left\lfloor\frac{1}{2}(\rho(u, t)-1)\right\rfloor<e,
$$

since $\rho(s, t)$ is odd. Taking the coefficient of $x^{e}$ in (45) yields $a_{e}+a_{e}^{*}=0$, as desired.

8.8. Corollary (Kalai, Klapper). Let $P$ be an Eulerian poset of odd rank $2 e+$ 1 , and let $\gamma$ and $\gamma^{*}$ be as in Corollary 8.3. Let

$$
\begin{aligned}
h(P, x) & =\gamma_{\hat{0} \hat{1}}(x)=h_{0}+h_{1} x+\cdots+h_{e} x^{e}, \\
h\left(P^{*}, x\right) & =\gamma_{\hat{1} \hat{0}}^{*}(x)=h_{0}^{*}+h_{1}^{*} x+\cdots+h_{e}^{*} x^{e} .
\end{aligned}
$$

Then $h_{e}=h_{e}^{*}$.

Proof. Using the notation of Proposition 8.7 with $s=\hat{0}$ and $t=\hat{1}$, we have $a_{e}=h_{e}$ and, by Corollary 8.3, $\tilde{\gamma}_{\hat{0} \hat{i}}=(-1)^{\rho(\hat{0}, \hat{1})} \gamma_{\hat{0} \hat{i}}^{*}$. Thus $a_{e}^{*}=(-1)^{\rho(\hat{0}, \hat{1})} h_{e}^{*}$. Now use Proposition 8.7 and the fact that $\rho(\hat{0}, \hat{1})$ is odd.

\section{ON a $q$-ANAlogue of Eulerian posets}

In this section we briefly discuss the possibility of developing a " $q$-analogue" of the theory of subdivisions of a simplex, or more generally, of formal subdivisions of an Eulerian poset. We discuss two natural $q$-analogues of Eulerian posets and show (Proposition 9.1) that they coincide. Unfortunately Proposition 9.1 also shows that these " $q$-Eulerian posets" are not nearly as prevalent as ordinary Eulerian posets. We do, however, have a (well-known) natural $q$-analogue $B_{d}(q)$ of the boolean algebra $B_{d}$, but we have been unable to construct a satisfactory theory of formal subdivisions of $B_{d}(q)$.

First consider the kernel $\lambda_{s t}(x)=(x-1)^{\rho(s, t)}$ on an Eulerian poset $P$. The "natural" $q$-analogue is given by

$$
\lambda_{s t}^{q}(x)=(x-1)(x-q) \cdots\left(x-q^{\rho(s, t)-1}\right) .
$$

The $q$-analogue of the boolean algebra $B_{d}$ is the lattice $B_{d}(q)$ of subspaces of a $d$-dimensional vector space over the finite field $\mathbf{F}_{q}$ (see [ $\mathrm{Sta}_{11}$, Example 3.10.2]). $B_{d}(q)$ is locally $q$-Eulerian (or even $q$-Eulerian, since it has a $\hat{0}$ and 
$\hat{1})$, in the sense that for all $s \leq t$ in $B_{d}(q)$,

$$
\mu_{s t}=(-1)^{m} q^{\left(\begin{array}{c}
m \\
2
\end{array}\right)},
$$

where $m=\rho(s, t)\left(\right.$ see [ $\mathrm{Sta}_{11}$, Example 3.10.2]). Note that $\lambda^{q}(x)$ is a $B_{d}(q)$ kernel, since the $q$-binomial theorem [ $\mathrm{Sta}_{11}$, Exercise 3.45] and the formula (47) imply that

$$
\lambda^{q}=\mu \bar{\zeta} \quad \text { in } I\left(B_{d}(q)\right) .
$$

(See Example 6.8.) The next result shows that the two notions of locally $q$ Eulerian given by equations (46) and (47) are equivalent, but that unfortunately $q$-Eulerian posets are much less prevalent than Eulerian posets.

9.1. Proposition. Let $q \geq 2$ be an integer, and let $P$ be a locally graded poset. The following three conditions are equivalent:

(a) $\lambda^{q}(x)$ (as defined by (46)) is a P-kernel.

(b) $\mu_{s t}=(-1)^{m} q^{\left(\begin{array}{c}m \\ 2\end{array}\right)}$ for all $s \leq t$ in $P$, where $m=\rho(s, t)$.

(c) For all $s \leq t$ in $P$ with $\rho(s, t)=m$, the number of elements $u \in[s, t]$ with $\rho(s, u)=i$ is equal to the q-binomial coefficient $\left(\begin{array}{c}\boldsymbol{m} \\ \boldsymbol{i}\end{array}\right)$. (See e.g. [ $\mathrm{Sta}_{11}$, p. 26] for the definition of $\left(\begin{array}{c}\boldsymbol{m} \\ \boldsymbol{i}\end{array}\right)$.)

Proof. (a) $\Rightarrow$ (c) Fix an interval $[s, t]$ of length $m$. Let $N_{i}$ be the number of $u \in[s, t]$ satisfying $\rho(s, u)=i$. Let

$$
\eta_{i}(x)=(x-1)(x-q) \cdots\left(x-q^{i-1}\right) \cdot(1-x)(1-q x) \cdots\left(1-q^{m-i-1} x\right) .
$$

Assume $\lambda^{q}(x)$ is a $P$-kernel. Thus by Theorem 6.5 we have $\bar{\lambda}^{q}=\left(\lambda^{q}\right)^{-1}$, which is equivalent to

$$
\sum_{i=0}^{m} N_{i} \eta_{i}(x)=\delta_{0 m}
$$

We have $N_{0}=1$. Set $x=q$ in (49). We obtain a linear equation in the variables $N_{0}$ and $N_{1}$, for which the coefficient of $N_{1}$ is nonzero, so $N_{1}$ is uniquely determined. Now set $x=q^{2}$ in (49). We obtain a linear equation in $N_{0}, N_{1}$, and $N_{2}$, for which the coefficient of $N_{2}$ is nonzero, so $N_{2}$ is uniquely determined. Continuing, we see that (49) uniquely determines $N_{0}, N_{1}, \ldots, N_{m-1}$ (and of course $N_{m}=1$ ). On the other hand, we know from (48) that $\lambda^{q}(x)$ is a kernel for $B_{d}(q)$ (where we choose $d \geq m$ ), so $N_{i}$ must be the number of elements of rank $i$ in an interval of length $m$ in $B_{d}(q)$. But this number is just $\left(\begin{array}{c}\boldsymbol{m} \\ \boldsymbol{i}\end{array}\right)$, proving (c).

(c) $\Rightarrow$ (b) Since $\mu_{s t}=(-1)^{m} q^{\left(\begin{array}{c}m \\ 2\end{array}\right)}$ for $B_{d}(q)$, we have (by the defining recurrence $\left[\mathrm{Sta}_{11}\right.$, Chapter 3.7,(14)] for $\mu$ ) that

$$
\sum_{i=0}^{m}\left(\begin{array}{c}
\boldsymbol{m} \\
\boldsymbol{i}
\end{array}\right)(-1)^{i} q^{\left(\frac{i}{2}\right)}=\delta_{0 m}
$$

But assuming (c), equation (50) implies that in fact $\mu_{s t}=(-1)^{m} q^{\left(\begin{array}{c}m \\ 2\end{array}\right)}$ for $P$. 
(b) $\Rightarrow$ (a) For any locally graded $P$, we have by Proposition 6.3 (or Example 6.8) that $\mu \bar{\zeta}$ is a $P$-kernel. But if (b) holds then by the $q$-binomial theorem [ Sta $_{11}$, Exercise 3.45] we have $\mu \bar{\zeta}=\lambda^{q}$.

It is natural to ask for a complete description of all posets $P$ satisfying the conditions of Proposition 9.1. For $\rho(P) \leq 2$ (where $\rho(P)$ denotes the rank of $P$ ) there is clearly a unique $P$ (up to isomorphism) for each $q \geq 2$. For $\rho(P)=$ $3, P$ is essentially a regular bipartite graph of degree $q+1$ with $2\left(q^{2}+q+1\right)$ vertices. Such graphs are clearly very numerous and have no special structure. For $\rho(P) \geq 4$ we suspect the posets $P$ are also quite numerous, though at the other extreme it is conceivable that for $\rho(P)$ sufficiently large (possibly $\rho(P) \geq 4) P$ must be isomorphic to $B_{d}(q)$ (so in particular $q$ is a prime power). As pointed out by M. Haiman, this unlikely result would yield an extremely simple axiomatization of finite projective spaces (of sufficiently large dimension).

Since $B_{d}(q)$ is a good $q$-analogue of $B_{d}$, we can ask whether the theory of subdivisions of $B_{d}$ (i.e., of the simplex $2^{V}$ with $\# V=d$ ) can be given a satisfactory $q$-analogue. The most obvious approach would be to extend the definition of formal subdivision of an Eulerian poset (Definition 7.4) to the poset $B_{d}(q)$. But, as discussed after the proof of Theorem 7.5, we do not see how to do so because the kernel $\lambda^{q}$ fails to satisfy $\lambda_{s u}^{q} \lambda_{u t}^{q}=\lambda_{s t}^{q}$ for $s \leq u \leq t$. A. Zelevinsky has suggested that one might need to change the incidence algebra $I\left(B_{d}\right)$ into a " $q$-incidence algebra" $I_{q}\left(B_{d}(q)\right)$, rather than an ordinary incidence algebra $I\left(B_{d}(q)\right)$.

\section{ACKNOWLEDGMENTS}

I am grateful to Lou Billera, Anders Björner, David Eisenbud, Mel Hochster, Gil Kalai, Bob MacPherson, Kei-ichi Watanabe, Sergey Yuzvinsky, Andrey Zelevinsky, and Günter Ziegler for helpful discusssions and suggestions. Special thanks go to Clara Chan for finding a serious error in my original version of $\S 4$.

\section{REFERENCES}

[Ba $\left.{ }_{1}\right]$ M. M. Bayer, The generalized Dehn-Sommerville equations revisited, Technical Report, Northeastern University, May, 1986.

$\left[\mathrm{Ba}_{2}\right] \longrightarrow$ Equidecomposable and weakly neighborly polytopes, Israel J. Math. (to appear).

[Bi] L. J. Billera, Polyhedral theory and commutative algebra, Mathematical Programming: The State of the Art (A. Bachem, M. Grötschel, and B. Korte, eds.), Springer-Verlag, BerlinHeidelberg-New York, 1983, pp. 57-77.

[Bj] A. Björner, Posets, regular CW complexes, and Bruhat order, European J. Combin. 5 (1984), 7-16.

$\left[\mathrm{Br}_{1}\right] \quad \mathrm{F}$. Brenti, Unimodal polynomials arising from symmetric functions, Proc. Amer. Math. Soc. 108 (1990), 1133-1141.

$\left[\mathrm{Br}_{2}\right] \longrightarrow$, Permutation enumeration, symmetric functions, and unimodality, Pacific J. Math., submitted.

[B-F-S] L. J. Billera, P. Filliman, and B. Sturmfels, Constructions and complexity of secondary polytopes, Adv. in Math. 83 (1990), 155-179. 
[B-K] M. M. Bayer and A. Klapper, A new index for polytopes, Discrete Comput. Geom. 6 (1991), 33-47.

[B-M] U. Betke and P. McMullen, Lattice points in lattice polytopes, Monatsh. Math. 99 (1985), 253-265.

[C] L. Comtet, Advanced combinatorics, Reidel, Dordrecht/Boston, 1974.

[Dan] V. I. Danilov, The geometry of toric varieties, Uspekhi Mat. Nauk. 33 (1978), 85-134; English transl. in Russian Math. Surveys 33 (1978), 97-154.

[Dav] R. J. Daverman, Decomposition of manifolds, Academic Press, New York, 1986.

[De] V. Deodhar, On some geometric aspects of Bruhat orderings, I. A fine decomposition of Bruhat cells, Invent. Math. 79 (1985), 499-511.

[G-K-Z] I. M. Gelfand, M. M. Kapranov, and A. V. Zelevinsky, Hyperdeterminants, Adv. Math. (to appear).

[G-M] M. Goresky and R. MacPherson, On the topology of complex algebraic sets, Algebraic Geometry (J.M. Aroca, et al., eds.), Lecture Notes in Math., vol. 961, Springer-Verlag, Berlin-Heidelberg-New York, 1982, pp. 119-129.

[G-Z-K] I. M. Gelfand, A. V. Zelevinsky, and M. M. Kapranov, Discriminants of polynomials in several variables and triangulations of Newton polyhedra, Leningrad Math. J. 2 (1991), 449-505.

[Ha] M. Haiman, A simple and relatively efficient triangulation of the $n$-cube, Discrete Comput. Geom. 6 (1991), 287-289.

[Hu] J. Humphreys, Reflection groups and Coxeter groups, Cambridge Univ. Press, Cambridge, 1990.

[H-S] P. J. Hilton and U. Stammbach, A course in homological algebra, Springer-Verlag, BerlinHeidelberg-New York, 1971.

[Ka] G. Kalai, The diameter of graphs of convex polytopes and $f$-vector theory, preprint.

[K1] V. Klee, A combinatorial analogue of Poincaré's duality theorem, Canadian J. Math. 16 (1964), 517-531.

[K-K] B. Kind and P. Kleinschmidt, Schälbare Cohen-Macauley[sic]-Komplexe und ihre Parametrisierung, Math. Z. 167 (1979), 173-179.

[K-L] D. Kazhdan and G. Lusztig, Representations of Coxeter groups and Hecke algebras, Invent. Math. 53 (1979), 165-184.

[L] C. Lee, Regular triangulations of convex polytopes, DIMACS Ser. in Discrete Math. and Theoret. Comput. Sci. (to appear).

[Ma $\left.{ }_{1}\right]$ I. G. Macdonald, Polynomials associated with finite cell-complexes, J. London Math. Soc. (2) 4 (1971), 181-192.

[ $\left.\mathrm{Ma}_{2}\right]$, Symmetric functions and hall polynomials, Oxford Univ. Press, Oxford, 1979.

[Mac] R. MacPherson, Intersection homology and perverse sheaves, preprint.

[Mc] P. McMullen, The maximum numbers of faces of a convex polytope, Mathematika 17 (1970), 179-184.

[Mi] M. Miyazaki, Characterization of Buchsbaum complexes, Manuscripta Math. 63 (1989), 245-254.

[Mu] J. R. Munkres, Elements of algebraic topology, Addison-Wesley, Menlo Park, CA, 1984.

[Sc] P. Schenzel, On the number of faces of simplicial complexes and the purity of Frobenius, Math. Z. 178 (1981), 125-142.

[Sp] E. H. Spanier, Algebraic topology, McGraw-Hill, New York, 1966.

[St $\left.{ }_{1}\right] \quad$ R. Stanley, Combinatorial reciprocity theorems, Adv. in Math. 14 (1974), 194-253.

$\left[\mathrm{Sta}_{2}\right] \quad$, The upper bound conjecture and Cohen-Macaulay rings, Stud. Appl. Math. 54 (1975), 135-142.

[Sta $\left.{ }_{3}\right]$ - Hilbert functions of graded algebras, Adv. in Math. 28 (1978), 57-83.

$\left[\mathrm{Sta}_{4}\right]$, Cohen-Macaulay complexes, in Higher combinatorics (M. Aigner, ed.), Reidel, Dordrecht-Boston, 1977, pp. 51-62. 
[Sta $\left.{ }_{5}\right] \quad$ Balanced Cohen-Macaulay complexes, Trans. Amer. Math. Soc. 249 (1979), 139157.

[Sta $\left.{ }_{6}\right]$, The number of faces of a simplicial convex polytope, Adv. in Math. 35 (1980), 236-238.

[Sta $\left.{ }_{7}\right]-$ Some aspects of groups acting on finite posets, J. Combin. Theory Ser. (A) 32 (1982), 132-161.

$\left[\mathrm{Sta}_{8}\right] \longrightarrow$, Combinatorics and commutative algebra, Progr. Math., vol. 41, Birkhäuser, Boston-Basel-Stuttgart, 1983.

[Sta $\left.{ }_{9}\right]$, The number of faces of simplicial polytopes and spheres, Discrete Geometry and Convexity (J.E. Goodman, et al., eds.), Ann. New York Acad. Sci., vol. 440, New York Acad. Sci., New York, 1985, pp. 212-223.

$\left[\mathrm{Sta}_{10}\right]-$ Generalized $h$-vectors, intersection cohomology of toric varieties, and related results, Commutative Algebra and Combinatorics (M. Nagata and H. Matsumura, eds.), Adv. Stud. Pure Math., vol. 11, Kinokuniya, Tokyo, and North-Holland, Amsterdam-New York, 1987, pp. 187-213.

[Sta $\left.{ }_{11}\right] \longrightarrow$, Enumerative combinatorics, Vol. 1, Wadsworth and Brooks/Cole, Pacific Grove, CA, 1986.

$\left[\mathrm{Sta}_{12}\right] \longrightarrow$, Unimodal and log-concave sequences in algebra, combinatorics, and geometry, Graph Theory and Its Applications: East and West, Ann. New York Acad. Sci., vol. 576, New York Acad. Sci., New York, 1989, pp. 500-535.

$\left[\mathrm{Sta}_{13}\right] \longrightarrow$ A monotonicity property of $h$-vectors and $h^{*}$-vectors, preprint.

[Ste] J. R. Stembridge, Eulerian numbers, tableaux, and the Betti numbers of a toric variety, Discrete Math. 99 (1992), 307-320.

[S-V] J. Stückrad and W. Vogel, Buchsbaum rings and applications, Springer-Verlag, BerlinHeidelberg-New York, 1986.

Abstract. In Part I a general theory of $f$-vectors of simplicial subdivisions (or triangulations) of simplicial complexes is developed, based on the concept of $l o$ cal $h$-vector. As an application, we prove that the $h$-vector of a Cohen-Macaulay complex increases under "quasi-geometric" subdivision, thus establishing a special case of a conjecture of Kalai and this author. Techniques include commutative algebra, homological algebra, and the intersection homology of toric varieties. In Part II we extend the work of Part I to more general situations. First a formal generalization of subdivision is given based on incidence algebras. Speciai cases are then developed, in particular one based on subdivisions of Eulerian posets and involving generalized $h$-vectors. Other cases deal with Kazhdan-Lusztig polynomials, Ehrhart polynomials, and a $q$-analogue of Eulerian posets. Many applications and examples are given throughout.

Department of Mathematics, Massachusetts Institute of Technology, Cambridge, MASSACHUSETTS 02139

E-mail address: rstan@math.mit.edu 